\title{
Kybernetika
}

\section{Zdenko Takáč}

OWA operators for discrete gradual intervals: implications to fuzzy intervals and multi-expert decision making

Kybernetika, Vol. 52 (2016), No. 3, 379-402

Persistent URL: http://dml.cz/dmlcz/145782

\section{Terms of use:}

(C) Institute of Information Theory and Automation AS CR, 2016

Institute of Mathematics of the Czech Academy of Sciences provides access to digitized documents strictly for personal use. Each copy of any part of this document must contain these Terms of use.

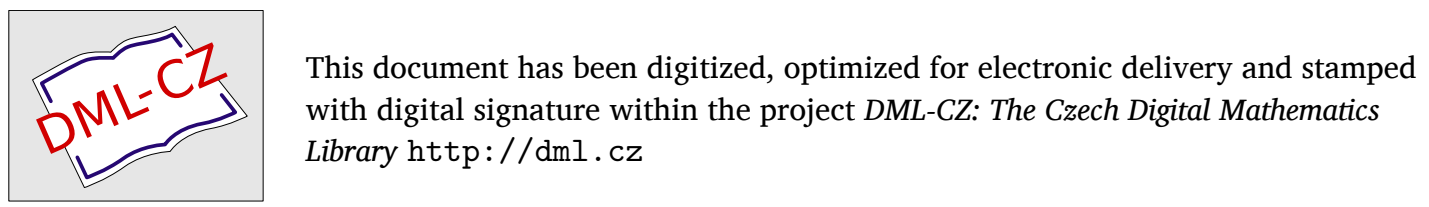




\title{
OWA OPERATORS FOR DISCRETE GRADUAL INTERVALS: IMPLICATIONS TO FUZZY INTERVALS AND MULTI-EXPERT DECISION MAKING
}

\author{
ZDENKO TAKÁČ
}

A new concept in fuzzy sets theory, namely that of gradual element, was introduced recently. It is known that the set of gradual real numbers is not ordered linearly. We restrict our attention to a discrete case and propose a class of linear orders for discrete gradual real numbers. Then, using idea of the so-called admissible order of intervals, we present a class of linear orders for discrete gradual intervals. Once we have the linear orders it is possible to define OWA operator for discrete gradual real numbers and OWA operator for discrete gradual intervals. Recall that gradual intervals also encompass fuzzy intervals, hence our results are applicable to the setting of fuzzy intervals. Our approach is illustrated on a multi-expert decision making problem.

Keywords: OWA operator, ordered weighted averaging operator, gradual number, gradual interval, fuzzy interval, linear order, total order, multi-expert decision making, type-2 fuzzy set

Classification: 03E72, 68T37

\section{INTRODUCTION}

In 30] Yager proposed an ordered weighted averaging operator (OWA for short) as follows: let $\mathbf{w}=\left(w_{1}, \ldots, w_{n}\right) \in[0,1]^{n}$ with $w_{1}+\ldots+w_{n}=1$ be a weighting vector, an OWA operator associated with $\mathbf{w}$ is a mapping $O W A_{\mathbf{w}}:[0,1]^{n} \rightarrow[0,1]$ defined by

$$
O W A_{\mathbf{w}}\left(x_{1}, \ldots, x_{n}\right)=\sum_{i=1}^{n} w_{i} x_{(i)}
$$

where $x_{(i)}$ denotes the $i$ th largest number among $x_{1}, \ldots, x_{n}$. This is one of the most widely used aggregation methods. It is of special significance in solving decision making problems.

Most of the existing OWA operators focus on aggregation of real numbers, however, there is a growing interest of scholars to study OWA operators on some more difficult structures. An OWA operator for fuzzy sets (type-1 OWA operator) is proposed in 31]

DOI: $10.14736 /$ kyb-2016-3-0379 
and more efficient $\alpha$-level approach to implementing the type-1 OWA operator is given in 32. Another point of view to OWA operators for fuzzy sets is proposed in [15, 23]. In [4] an OWA operator for intervals (IVOWA) is proposed, and there is proved that it is a special case of the type-1 OWA operator. Another approach to OWA operator for intervals is given in 29 .

The distinction between fuzziness and imprecision is discussed in 9 ] and [10] which leads to proposing a new concept in fuzzy sets theory, namely a gradual element. A gradual real number possess fuzziness but not uncertainty, instead of what is debatable called 'fuzzy number' (we will refer to as fuzzy interval, see [20, 28]) which possess both. A gradual interval is simply a crisp interval of gradual real numbers. This is also a new way of looking at fuzzy intervals [16]: instead of considering them as fuzzy sets, one can see them as crisp intervals of gradual real numbers. Note that the similar point of view on gradualness is presented in [14, 25] and [17.

The aim of this paper is to propose an OWA operator for gradual numbers and gradual intervals. The crucial point of this is the existence of a linear order. As is stated in [10] the set of gradual numbers is not linearly ordered and the same holds for the set of gradual intervals. Hence our first objection is to introduce some linear order on these two sets. This is of course only possible in discrete case, thus we first introduce a linear order on the set of all discrete gradual real numbers. Then, based on the idea of admissible orders [3, we propose a linear order on the set of all discrete gradual intervals. Having these linear orders we can proceed to the definition of OWA operator for discrete gradual real numbers (DGOWA) and for discrete gradual intervals (DGIOWA).

There are two essential differences between our DGIOWA and OWA operators defined in 31] and [15]: 1. the weights of later are fuzzy sets whereas the weights of former are gradual numbers; 2 . the former operates on the set of all discrete gradual intervals in $[0,1]$ whereas the later operates on the set of all normal convex fuzzy sets in $[0,1]$ which is in a discrete case the proper subset of the set of all discrete gradual intervals, Hence, from this point of view the DGIOWA is more general than the existing OWA operators. In some situations it is more natural to represent information by a gradual interval (which is not a fuzzy set) than by a fuzzy set. For instance, in multi-expert decision making problem, a decision maker has more freedom in assessment of alternatives in case of using gradual intervals and subsequent application of DGIOWA. We discuss this issue in Section 4.

It is worth pointing out that we also show the relation between partial order of gradual intervals and partial order of fuzzy intervals (fuzzy truth values) used in the type-2 fuzzy sets setting (see e. g. [5, 18]).

We apply proposed linear order and DGIOWA operator in a multi-expert decision making (MEDM) problem. An MEDM problem 24 can be described as follows: we have a set of alternatives, a set of experts, and each expert provides a preference on the set of alternatives. A decision maker (DM) is looking for the best alternative. A general model for solving an MEDM problem is introduced in [6, 12]:

1. Making the information uniform. It is not our purpose to study this point. We assume that the experts' preferences are uniform, represented by utility functions (in our case functions that associate each alternative with a discrete gradual interval). 
2. Application of a selection process, which is applied in two steps [2]:

- Aggregation phase: the aim is to combine the individual preferences to obtain a collective preference of each alternative. We apply our DGIOWA operator in this phase.

- Exploitation phase: the aim is to obtain an ordering of the collective preferences, and to choose the best alternative(s). We apply our linear order for discrete gradual intervals in this phase.

The paper is organized as follows. In Section 2 we introduce a linear order on the set of all discrete gradual real numbers and define an OWA operator on this set. In Section 3 we discuss the relation between gradual intervals and fuzzy intervals, then introduce a linear order and OWA operator on the set of all discrete gradual intervals. Section 4 presents an application of proposed OWA operators and linear orders to real world MEDM problem. The conclusions are discussed in Section 5.

\section{OWA OPERATORS FOR DISCRETE GRADUAL REAL NUMBERS}

The aim of this section is to propose OWA operator for discrete gradual real numbers.

\subsection{Discrete gradual real numbers}

Definition 2.1. (Dubois and Prade [9, Fortin et al. [10]) A gradual real number $\breve{r}$ is defined by an assignment function $B_{\breve{r}}:(0,1] \rightarrow \mathbb{R}$. The set of all gradual real numbers is denoted by $G(\mathbb{R})$.

In this article we consider discrete gradual real numbers $B_{\breve{r}}:\left\{\alpha_{1}, \ldots, \alpha_{k}\right\} \rightarrow \mathbb{R}$, where $0<\alpha_{1}<\alpha_{2}<\ldots<\alpha_{k}=1$ (mostly, $\alpha_{i}=\frac{i}{k}$ ). For given $k$ and $0<\alpha_{1}<\alpha_{2}<$ $\ldots<\alpha_{k}=1$, the set of all discrete gradual real numbers is denoted by $D G_{k}(\mathbb{R})$. For simplicity we do not distinguish between gradual number and its assignment function, thus we write $\breve{r}(\alpha)$ instead of $B_{\breve{r}}(\alpha)$. We say that $\breve{r}, \breve{s} \in D G_{k}(\mathbb{R})$ are equal, write $\breve{r}=\breve{s}$, if $\breve{r}\left(\alpha_{i}\right)=\breve{s}\left(\alpha_{i}\right)$ for all $i=1, \ldots, k$.

Gradual numbers encompass real numbers: for every $r \in \mathbb{R}$ there exists $\breve{r} \in D G_{k}(\mathbb{R})$ such that $\breve{r}\left(\alpha_{i}\right)=r$ for all $i=1, \ldots, k$. We will write $\breve{r} \stackrel{\circ}{=} r$ to emphasize that $r$ and $\breve{r}$ are objects of different kinds. For instance, instead of $\breve{r}\left(\alpha_{i}\right)=1$ for all $i=1, \ldots, k$ we will write $\breve{r} \stackrel{\circ}{=}$, or simply $\breve{1}$.

\subsection{Extended aggregation functions}

Definition 2.2. A function $A^{(n)}:[0,1]^{n} \rightarrow[0,1]$ is called an $n$-ary aggregation function on $[0,1]$ if it satisfies the conditions:

(1) $A^{(n)}(0, \ldots, 0)=0$ and $A^{(n)}(1, \ldots, 1)=1$;

(2) $A^{(n)}$ is nondecreasing in each variable;

(3) $A^{(1)}(x)=x$ for all $x \in[0,1]$. 
Definition 2.3. (Grabisch et al. [1]) A mapping $\mathbb{A}: \bigcup_{n \in \mathbb{N}}[0,1]^{n} \rightarrow[0,1]$ is called an extended aggregation function on $\bigcup_{n \in \mathbb{N}}[0,1]^{n}$ if its restriction $A^{(n)}:=\mathbb{A}_{[0,1]^{n}}$ to $[0,1]^{n}$ is an aggregation function on $[0,1]^{n}$ for all $n \in \mathbb{N}$.

We will sometimes use symbol $\mathbb{A}_{\mathbf{v}}$ instead of $\mathbb{A}$ to emphasize that extended aggregation function may (not necessarily) depend on a weighting vector $\mathbf{v}$, e.g. weighted averages.

Definition 2.4. We say that an extended aggregation operator $\mathbb{A}_{\mathbf{v}}$ satisfies property $(\mathrm{P} 1)$ for a fixed $k \in \mathbb{N}$ if:

For all $\left(x_{1}, \ldots, x_{k}\right),\left(y_{1}, \ldots, y_{k}\right) \in[0,1]^{k}$ it holds

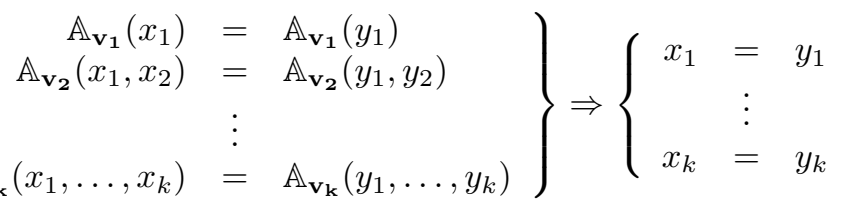

where $\mathbf{v}_{\mathbf{1}}=\left(v_{11}\right), \ldots, \mathbf{v}_{\mathbf{k}}=\left(v_{k 1}, \ldots, v_{k k}\right)$ are any weighting vectors with $v_{i j} \neq 0$ for all $i=1, \ldots, k, j=1, \ldots, i$.

Remark 2.5. Examples of extended aggregation functions which do not satisfy property (P1) are e.g. maximum and minimum. On the other hand, typical function which satisfies the property is arithmetic mean, or more generally, any weighted average with non-zero weights.

If $I=\left\{i_{1}, \ldots, i_{m}\right\}$ is a finite index set, we will write shortly $\mathbb{A}\left(x_{i}\right)_{i \in I}$ instead of $\mathbb{A}\left(x_{i_{1}}, \ldots, x_{i_{m}}\right)$.

\subsection{Linear order of discrete gradual real numbers}

We propose a linear order of discrete gradual real numbers in this section.

Our order (comparison of $\breve{r}$ and $\breve{s}$ ) is based on the comparison of extended aggregation functions (EAFs, for short) $\mathbb{A}_{\mathbf{v}}$ of $\breve{r}\left(\alpha_{1}\right), \ldots, \breve{r}\left(\alpha_{k}\right)$ and $\breve{s}\left(\alpha_{1}\right), \ldots, \breve{s}\left(\alpha_{k}\right)$ (step 2 of the following algorithm) w.r.t. given weighting vector $\mathbf{v}$ (even if $\mathbb{A}_{\mathbf{v}}$ does not depend on $\mathbf{v}$ ). If the EAFs are equal, we omit the set of all elements with minimal weight (step 3), redistribute the sum of their weights to the rest of the elements (step 5, we discuss the technique of redistribution below the algorithm) and compare EAFs again (step 2). We repeat these steps till we get a strict inequality or we omit all the elements. If the later is true, we refine our procedure as follows: we always omit exactly one element - the element corresponding to minimal $\alpha_{i}$ among the elements with minimal weight (step 4), redistribute its weight to the rest of the elements (step 5) and compare EAFs (step 2). We repeat these steps till we get a strict inequality or we omit all the elements. 


\section{Algorithm 1.}

Let $\mathbb{A}_{\mathbf{v}}: \bigcup_{n \in \mathbb{N}}[0,1]^{n} \rightarrow[0,1]$ be an extended aggregation function, $\breve{r}, \breve{s} \in D G_{k}(\mathbb{R})$ be discrete gradual real numbers, $\mathbf{v}=\left(v_{1}, \ldots, v_{k}\right) \in[0,1]^{k}$ be a weighting vector with $v_{1}+\ldots+v_{k}=1$ and $g:\{1,2, \ldots, k\} \rightarrow[0,1]$ be a function with appropriate properties specified in the algorithm.

1. Initialization:

$$
\begin{aligned}
& I:=\{1, \ldots, k\} . \\
& M:=\emptyset . \\
& s:=1 . \\
& v_{i}^{\prime}:=v_{i} \text { for all } i \in I .
\end{aligned}
$$

2. Comparison:

If $\mathbb{A}_{\mathbf{v}^{\prime}}\left(\breve{r}\left(\alpha_{i}\right)\right)_{i \in I}<\mathbb{A}_{\mathbf{v}^{\prime}}\left(\breve{s}\left(\alpha_{i}\right)\right)_{i \in I}$, then $\breve{r} \prec_{\mathbb{A}, \mathbf{v}, g} \breve{s}$ (END).

If $\mathbb{A}_{\mathbf{v}^{\prime}}\left(\breve{r}\left(\alpha_{i}\right)\right)_{i \in I}>\mathbb{A}_{\mathbf{v}^{\prime}}\left(\breve{s}\left(\alpha_{i}\right)\right)_{i \in I}$, then $\breve{s} \prec_{\mathbb{A}, \mathbf{v}, g} \breve{r}$ (END).

If $s=1$, then go to step 3 .

If $s=2$, then go to step 4 .

\section{Omitting 1:}

$M:=M \cup\left\{j \mid v_{j}=\min \left\{v_{i} \mid i \in I\right\}\right\}$.

$I:=I-M$.

If $I \neq \emptyset$, then go to step 5 , else $M:=\emptyset, I:=\{1, \ldots, k\}, s:=2$.

4. Omitting 2:

$M:=M \cup \min \left\{j \mid v_{j}=\min \left\{v_{i} \mid i \in I\right\}\right\}$.

$I:=I-M$.

If $I=\emptyset$, then $\breve{r} \approx \breve{s}$ (END).

5. Redistribution of weights:

$\mathbf{v}^{\prime}:=\left(v_{1}^{\prime}, \ldots, v_{k}^{\prime}\right)$, where $v_{i}^{\prime}:=v_{i}+g(i)$ for all $i \in I$, and $g:\{1,2, \ldots, k\} \rightarrow[0,1]$ is a function with $\sum_{i \in I} v_{i}^{\prime}=1$.

Go to step 2.

Remark 2.6. Algorithm 1 is a generalization of algorithm given in 27] where only weighted averages were used in step 2 (Comparison). For more detailed explanation of algorithm see above mentioned paper.

Remark 2.7. Redistribution of weights of the omitted elements is done by a redistribution function $g(i)$ in the following way: $v_{i}^{\prime}=v_{i}+g(i)$, where $g(i) \geq 0$ for all $i$. The redistribution function $g$ should satisfy $\sum_{i \in I} g(i)=\sum_{j \in M} v_{j}$ which clearly forces $\sum_{i \in I} v_{i}^{\prime}=1$ (the sum of all new weights is equal to 1). For instance, $g(i)=\frac{v_{s}}{k-|M|}$ where $|M|$ denotes the cardinality of $M, v_{s}=\sum_{j \in M} v_{j}$, i. e., $v_{i}^{\prime}=v_{i}+\frac{v_{s}}{k-|M|}$ (the weight of each element is increased by the same value); or $g(i)=\frac{v_{i}}{1-v_{s}} \cdot v_{s}$, i. e., $v_{i}^{\prime}=v_{i}+\frac{v_{i}}{1-v_{s}} \cdot v_{s}$ (the weights of individual elements are increased in proportion of the original weights). 
Theorem 2.8. Let $\prec_{\mathbb{A}, \mathbf{v}, g}$ be given by Algorithm 1 for some fixed redistribution function $g$, weighting vector $\mathbf{v}=\left(v_{1}, \ldots, v_{k}\right) \in(0,1]^{k}$ with $v_{1}+\ldots+v_{k}=1$ and extended aggregation function $\mathbb{A}_{\mathbf{v}}$ satisfying property (P1). A binary relation $\preceq_{\mathbb{A}, \mathbf{v}, g}$ defined by

$$
\breve{r} \preceq_{\mathbb{A}, \mathbf{v}, g} \breve{s} \quad \text { iff } \quad\left(\breve{r} \prec_{\mathbb{A}, \mathbf{v}, g} \breve{s} \quad \text { or } \quad \breve{r}=\breve{s}\right)
$$

is a linear order on the set of all discrete gradual real numbers.

P r o of. It is obvious that $\preceq_{\mathbb{A}, \mathbf{v}, g}$ is a partial order on the set of all gradual real numbers. Hence it is sufficient to show that each pair $\breve{r}, \breve{s}$ of gradual real numbers is comparable by $\preceq_{\mathbb{A}, \mathbf{v}, g}$. From Algorithm 1 we obtain $\breve{r} \prec_{\mathbb{A}, \mathbf{v}, g} \breve{s}, \breve{s} \prec_{\mathbb{A}, \mathbf{v}, g} \breve{r}$, or

$$
\begin{array}{rlrl}
\mathbb{A}_{\mathbf{v}_{\mathbf{1}}}\left(\breve{r}\left(\alpha_{i}\right)\right)_{i \in I_{1}} & =\mathbb{A}_{\mathbf{v}_{\mathbf{1}}}\left(\breve{s}\left(\alpha_{i}\right)\right)_{i \in I_{1}}, & & I_{1}=\{1,2, \ldots, k\}, \\
\mathbb{A}_{\mathbf{v}_{\mathbf{2}}}\left(\breve{r}\left(\alpha_{i}\right)\right)_{i \in I_{2}} & =\mathbb{A}_{\mathbf{v}_{\mathbf{2}}}\left(\breve{s}\left(\alpha_{i}\right)\right)_{i \in I_{2}}, & & I_{2} \subseteq I_{1},\left|I_{2}\right|=k-1, \\
& \vdots & & \\
\mathbb{A}_{\mathbf{v}_{\mathbf{k}}}\left(\breve{r}\left(\alpha_{i}\right)\right)_{i \in I_{k}}=\mathbb{A}_{\mathbf{v}_{\mathbf{k}}}\left(\breve{s}\left(\alpha_{i}\right)\right)_{i \in I_{k}}, & & I_{k} \subseteq I_{k-1},\left|I_{k}\right|=1,
\end{array}
$$

for some weighting vectors $\mathbf{v}_{\mathbf{1}}, \ldots, \mathbf{v}_{\mathbf{k}}$ with non-zero coordinates. Hence, by property (P1) it follows that $\breve{r}\left(\alpha_{i}\right)=\breve{s}\left(\alpha_{i}\right)$ for all $i=1, \ldots, k$, which gives $\breve{r}=\breve{s}$.

It is clear that $\preceq_{\mathbb{A}, \mathbf{v}, g}$ refines partial order of gradual real numbers [10]: $\breve{r} \leq \breve{s}$ iff $\breve{r}\left(\alpha_{i}\right) \leq \breve{s}\left(\alpha_{i}\right)$ for all $i=1, \ldots, k$. This means that, for all extended aggregation functions $\mathbb{A}$, weighting vectors $\mathbf{v}$ and redistribution functions $g$ it holds:

$$
\breve{r} \preceq_{\mathbb{A}, \mathbf{v}, g} \breve{s} \quad \text { whenever } \quad \breve{r} \leq \breve{s} \text {. }
$$

Moreover, our linear order $\preceq_{\mathbb{A}, \mathbf{v}, g}$ generalizes the 'standard' order of real numbers $\leq$, i.e.,

$$
\breve{r} \preceq_{\mathbb{A}, \mathbf{v}, g} \breve{s} \quad \text { iff } \quad r \leq s
$$

for all $r, s \in R$ where $\breve{r} \stackrel{\circ}{=}$ and $\breve{s} \stackrel{\circ}{=} s$.

Example 1. Let extended aggregation function $\mathbb{A}_{\mathbf{v}}$ be a weighted average, weighting vector $\mathbf{v}$ be given by the fifth column of Table 1 . discrete gradual real numbers $\breve{r}, \breve{s}$ (see Figure 1 on the left) by the third and fourth columns, and let redistribution function be $g(i)=\frac{v_{s}}{k-|M|}$. Then $\breve{r} \prec_{\mathbb{A}, \mathbf{v}, g} \breve{s}$ (the detailed application of Algorithm 1 is showed in Table 1).

Remark 2.9. Although gradual numbers are functions from (0.1] to real numbers, we depict them in a reversed form, i. e. independent variable $\alpha_{i} \in(0,1]$ is on the vertical axis and dependent variables $\breve{r}\left(\alpha_{i}\right), \breve{s}\left(\alpha_{i}\right)$ are on the horizontal axis. Note that we work with discrete gradual numbers, hence only the points are important and the lines which connect the points are added just for better lucidity. 


\begin{tabular}{c|c|cc|c|ccccc}
$i$ & $\alpha_{i}$ & $\breve{r}\left(\alpha_{i}\right)$ & $\breve{s}\left(\alpha_{i}\right)$ & $v_{i}$ & $v_{i}^{\prime}$ & $v_{i}^{\prime}$ & $v_{i}^{\prime}$ & $v_{i}^{\prime}$ & $v_{i}^{\prime}$ \\
\hline 10 & 1 & 3 & 2 & 0.14 & $0.18 \overline{6}$ & $0 . \overline{3}$ & $0.14 \overline{7}$ & 0.1575 & 0.17 \\
9 & 0.9 & 3 & 3 & 0.14 & $0.18 \overline{6}$ & $0 . \overline{3}$ & $0.14 \overline{7}$ & 0.1575 & 0.17 \\
8 & 0.8 & 3 & 4 & 0.14 & $0.18 \overline{6}$ & $0 . \overline{3}$ & $0.14 \overline{7}$ & 0.1575 & 0.17 \\
7 & 0.7 & 4 & 5 & 0.1 & $0.14 \overline{6}$ & & $0.10 \overline{7}$ & 0.1175 & 0.13 \\
6 & 0.6 & 4 & 4 & 0.1 & $0.14 \overline{6}$ & & $0.10 \overline{7}$ & 0.1175 & 0.13 \\
5 & 0.5 & 4 & 3 & 0.1 & $0.14 \overline{6}$ & & $0.10 \overline{7}$ & 0.1175 & 0.13 \\
4 & 0.4 & 3 & 4 & 0.07 & & & $0.0 \overline{7}$ & 0.0875 & 0.1 \\
3 & 0.3 & 3 & 2 & 0.07 & & & $0.0 \overline{7}$ & 0.0875 & \\
2 & 0.2 & 2 & 2 & 0.07 & & & $0.0 \overline{7}$ & & \\
1 & 0.1 & 3 & 3 & 0.07 & & & & & \\
\hline$W A(\breve{r})$ & & & & 3.23 & 3.44 & 3 & $3.24 \overline{5}$ & 3.3525 & $\mathbf{3 . 3 9}$ \\
$W A(\breve{s})$ & & & & 3.23 & 3.44 & 3 & $3.24 \overline{5}$ & 3.3525 & $\mathbf{3 . 4 9}$ \\
order & & & & $?$ & $?$ & $?$ & $?$ & $?$ & $\breve{r} \prec \mathbb{A}, \mathbf{v}, g$ \\
\end{tabular}

Tab. 1. Application of Algorithm 1 (Example 1). By $W A(\breve{r})$ we denote weighted average of $\breve{r}\left(\alpha_{i}\right) \mathrm{s}$.

Example 2. It is easy to check that the linear order $\preceq_{\mathbb{A}, \mathbf{v}, g}$ depends on weighting vector $\mathbf{v}$. We will now show that it also depends on redistribution function $g$. Let extended aggregation function $\mathbb{A}_{\mathbf{v}}$ be a weighted average, weighting vector $\mathbf{v}$ be given by the fifth column of Table 2 , discrete gradual real numbers $\breve{r}, \breve{s}$ by the third and fourth columns, and let redistribution functions be $g_{1}(i)=\frac{v_{i}}{1-v_{s}} \cdot v_{s}$ (the sixth and seventh columns) and $g_{2}(i)=\frac{v_{s}}{k-|M|}$ (the eighth column). Then $\breve{s} \prec_{\mathbb{A}, \mathbf{v}, g_{1}} \breve{r}$ and $\breve{r} \prec_{\mathbb{A}, \mathbf{v}, g_{2}} \breve{s}$ (see Table 2).

\begin{tabular}{c|c|cc|c|cc|c}
$i$ & $\alpha_{i}$ & $\breve{r}\left(\alpha_{i}\right)$ & $\breve{s}\left(\alpha_{i}\right)$ & $v_{i}$ & $v_{i}^{\prime}\left(g_{1}\right)$ & $v_{i}^{\prime}\left(g_{1}\right)$ & $v_{i}^{\prime}\left(g_{2}\right)$ \\
\hline 10 & 1 & 5 & 2 & 0.14 & 0.1591 & 0.1944 & 0.155 \\
9 & 0.9 & 5 & 2 & 0.14 & 0.1591 & 0.1944 & 0.155 \\
8 & 0.8 & 4 & 3 & 0.12 & 0.1364 & $0.1 \overline{6}$ & 0.135 \\
7 & 0.7 & 4 & 3 & 0.12 & 0.1364 & $0.1 \overline{6}$ & 0.135 \\
6 & 0.6 & 3 & 5 & 0.1 & 0.1136 & 0.1389 & 0.115 \\
5 & 0.5 & 2 & 6 & 0.1 & 0.1136 & 0.1389 & 0.115 \\
4 & 0.4 & 2 & 5 & 0.08 & 0.0909 & & 0.095 \\
3 & 0.3 & 2 & 5 & 0.08 & 0.0909 & & 0.095 \\
2 & 0.2 & 3 & 4 & 0.06 & & & \\
1 & 0.1 & 3 & 2 & 0.06 & & & \\
\hline$W A(\breve{r})$ & & & & 3.54 & 3.6136 & $\mathbf{3 . 9 7 2 2}$ & $\mathbf{3 . 5 8 5}$ \\
$W A(\breve{s})$ & & & & 3.54 & 3.6136 & $\mathbf{3 . 3 0 5 6}$ & $\mathbf{3 . 6 4 5}$ \\
order & & & & $?$ & $?$ & $\breve{s} \prec_{\mathbb{A}, \mathbf{v}, g_{1}} \breve{r}$ & $\breve{r} \prec_{\mathbb{A}, \mathbf{v}, g_{2}} \breve{s}$
\end{tabular}

Tab. 2. Application of Algorithm 1 (Example 2). By $v_{i}^{\prime}\left(g_{1}\right)$ and $v_{i}^{\prime}\left(g_{2}\right)$ are denoted weights calculated via redistribution functions $g_{1}$ and $g_{2}$, respectively. 

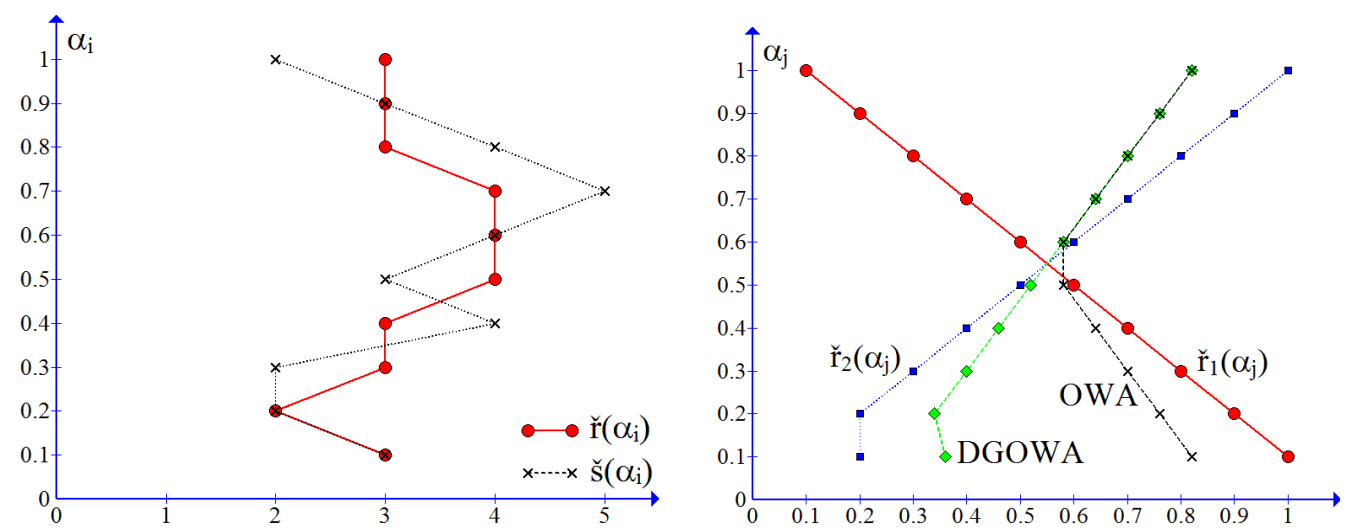

Fig. 1. See Example 1 (left) or Example 3 (right).

\subsection{OWA operators for discrete gradual real numbers}

Since we have a linear order, we can define an OWA operator for discrete gradual numbers. Instead of using real numbers we employ gradual numbers as weights.

Note that the sum of discrete gradual real numbers $\breve{w}_{1}, \breve{w}_{2} \in D G_{k}(\mathbb{R})$ is given by $\left(\breve{w}_{1}+\breve{w}_{2}\right)\left(\alpha_{i}\right)=\breve{w}_{1}\left(\alpha_{i}\right)+\breve{w}_{2}\left(\alpha_{i}\right)$ for all $i=1, \ldots, k$.

Definition 2.10. Let $\breve{\mathbf{w}}=\left(\breve{w}_{1}, \ldots, \breve{w}_{n}\right) \in D G_{k}([0,1])^{n}$ with $\breve{w}_{1}+\ldots+\breve{w}_{n}=\breve{1}$ be a weighting vector of discrete gradual numbers. A discrete gradual numbers OWA operator (DGOWA for short) associated with $\breve{\mathbf{w}}$ is a mapping $D G O W A_{\breve{\mathbf{w}}, \mathbf{v}, g}^{\preceq}: D G_{k}(\mathbb{R})^{n} \rightarrow$ $D G_{k}(\mathbb{R})$ defined by

$$
\operatorname{DGOW} A_{\breve{\mathbf{w}}}^{\breve{\mathbb{A}}, \mathbf{v}, g}\left(\breve{r}_{1}, \ldots, \breve{r}_{n}\right)=\sum_{i=1}^{n} \breve{w}_{i} \breve{r}_{(i)},
$$

where $\breve{r}_{(i)}, i=1, \ldots, n$, denote the $i$ th greatest component of the input $\left(\breve{r}_{1}, \ldots, \breve{r}_{n}\right)$ with respect to a linear order $\preceq_{\mathbb{A}, \mathbf{v}, g}$.

Application of the gradual number arithmetic [10] to equation (3) leads to computation of $k$ independent results:

$$
\operatorname{DGOW} A_{\breve{\mathbf{w}}}^{\mathfrak{\mathbb { A }}_{\mathbf{w}, \mathbf{v}, g}}\left(\breve{r}_{1}, \ldots, \breve{r}_{n}\right)\left(\alpha_{j}\right)=\sum_{i=1}^{n} \breve{w}_{i}\left(\alpha_{j}\right) \breve{r}_{(i)}\left(\alpha_{j}\right)
$$

for all $j=1, \ldots, k$. This means that DGOWA operator is very easily computable.

Obviously, the proposed DGOWA operator can also be used with real weights $w_{1}, \ldots, w_{n}$. Moreover, the following theorem shows that our operator encompasses 'standard' OWA operator for real numbers. 
Theorem 2.11. Let $\mathbf{w}=\left(w_{1}, \ldots, w_{n}\right) \in[0,1]^{n}$ satisfy $w_{1}+\ldots+w_{n}=1$, and $r_{1}, \ldots, r_{n} \in$ $\mathbb{R}$. Let $\breve{w}_{i} \stackrel{\circ}{=} w_{i}, \breve{r}_{i} \stackrel{\circ}{=} r_{i}$, for $i=1, \ldots, n$, be discrete gradual real numbers, and $\breve{\mathbf{w}}=\left(\breve{w}_{1}, \ldots, \breve{w}_{n}\right)$. Then

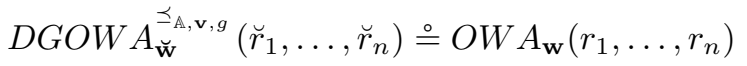

for any linear order $\preceq_{\mathbb{A}, \mathbf{v}, g}$.

Proof. The proof starts with the observation that $r_{(n)} \leq \ldots \leq r_{(1)}$ implies $\breve{r}_{(n)} \preceq_{\mathbb{A}, \mathbf{v}, g}$ $\ldots \preceq_{\mathbb{A}, \mathbf{v}, g} \breve{r}_{(1)}$ for any linear order $\preceq_{\mathbb{A}, \mathbf{v}, g}$. Then

$$
D G O W A_{\breve{\mathbf{w}}}^{\preceq_{\mathbb{A}, \mathbf{v}, g}}\left(\breve{r}_{1}, \ldots, \breve{r}_{n}\right)=\sum_{i=1}^{n} \breve{w}_{i} \breve{r}_{(i)} \stackrel{\circ}{=} \sum_{i=1}^{n} w_{i} r_{(i)}=O W A_{\mathbf{w}}\left(r_{1}, \ldots, r_{n}\right) .
$$

It is worth pointing out that, in general, DGOWA operator cannot be represented by $k$ OWA operators in the following form:

$$
D G O W A_{\left(\breve{w}_{1}, \ldots, \breve{w}_{n}\right)}^{\Upsilon_{\mathbb{A}, g}}\left(\breve{r}_{1}, \ldots, \breve{r}_{n}\right)\left(\alpha_{j}\right)=O W A_{\left(\breve{w}_{1}\left(\alpha_{j}\right), \ldots, \breve{w}_{n}\left(\alpha_{j}\right)\right)}\left(\breve{r}_{1}\left(\alpha_{j}\right), \ldots, \breve{r}_{n}\left(\alpha_{j}\right)\right)
$$

for all $j=1, \ldots, k$. The reason is that the $i$ th greatest element of $\breve{r}_{1}, \ldots, \breve{r}_{n}$ with respect to $\preceq_{\mathbb{A}, \mathbf{v}, g}$ need not correspond to the $i$ th greatest element of $\breve{r}_{1}\left(\alpha_{j}\right), \ldots, \breve{r}_{n}\left(\alpha_{j}\right)$ with respect to the 'standard' order of real numbers. We next give a counterexample.

Example 3. Let discrete gradual real numbers $\breve{r}_{1}, \breve{r}_{2}$ be given by Table 3 , see Figure 1 (on the right), and let $\mathbb{A}$ be weighted average. Clearly, $\breve{r}_{1} \preceq_{\mathbb{A}, \mathbf{v}, g}{\breve{r_{2}}}_{\text {for }}$ weighting vector $\mathbf{v}=(0.1,0.1, \ldots, 0.1)$. We can see that $D G O W A_{(0.8,0.2)}^{\mathfrak{A}_{\mathbb{A}, \mathbf{v}, g}}\left(\breve{r}_{1}, \breve{r}_{2}\right)\left(\alpha_{j}\right)$ is not equal to $O W A_{(0.8,0.2)}\left(\breve{r}_{1}\left(\alpha_{j}\right), \breve{r}_{2}\left(\alpha_{j}\right)\right)$ for $j \in\{1, \ldots, 5\}$. The difference lays in the fact that in DGOWA the greater weight 0.8 is assigned to the greater element $\breve{r}_{2}$ for all $\alpha_{j}$, however, in OWA operator the greater weight is assigned to the greater number of the pair $\breve{r}_{1}\left(\alpha_{j}\right), \breve{r}_{2}\left(\alpha_{j}\right)$ which is $\breve{r}_{1}\left(\alpha_{j}\right)$ for $j=1, \ldots, 5$, and $\breve{r}_{2}\left(\alpha_{j}\right)$ for $j=6, \ldots, 10$ :

$$
\operatorname{DGOW} A_{(0.8,0.2)}^{\preceq_{\mathbb{A}, \mathbf{v}, g}}\left(\breve{r}_{1}, \breve{r}_{2}\right)\left(\alpha_{j}\right)=0.8 \cdot \breve{r}_{2}\left(\alpha_{j}\right)+0.2 \cdot \breve{r}_{1}\left(\alpha_{j}\right)
$$

$$
O W A_{(0.8,0.2)}\left(\breve{r}_{1}\left(\alpha_{j}\right), \breve{r}_{2}\left(\alpha_{j}\right)\right)=0.8 \cdot \max \left\{\breve{r}_{1}\left(\alpha_{j}\right), \breve{r}_{2}\left(\alpha_{j}\right)\right\}+0.2 \cdot \min \left\{\breve{r}_{1}\left(\alpha_{j}\right), \breve{r}_{2}\left(\alpha_{j}\right)\right\}
$$

For complete results see Table 3 and Figure 1 . Recall that the difference disappears if $\breve{r}_{1} \leq \breve{r_{2}}$.

\section{OWA OPERATORS FOR DISCRETE GRADUAL INTERVALS}

In this section we extend the results of previous section to the setting of discrete gradual intervals (which encompasses also fuzzy intervals). First, gradual intervals and aggregation functions for gradual numbers are described, then linear order of discrete gradual intervals is proposed, and finally, OWA operator for discrete gradual intervals is defined and discussed. 


\begin{tabular}{c|c|cc|c|c}
$j$ & $\alpha_{j}$ & $\breve{r}_{1}\left(\alpha_{j}\right)$ & $\breve{r}_{2}\left(\alpha_{j}\right)$ & $D G O W A$ & $O W A$ \\
\hline 10 & 1 & 0.1 & 1 & 0.82 & 0.82 \\
9 & 0.9 & 0.2 & 0.9 & 0.76 & 0.76 \\
8 & 0.8 & 0.3 & 0.8 & 0.7 & 0.7 \\
7 & 0.7 & 0.4 & 0.7 & 0.64 & 0.64 \\
6 & 0.6 & 0.5 & 0.6 & 0.58 & 0.58 \\
5 & 0.5 & 0.6 & 0.5 & 0.52 & 0.58 \\
4 & 0.4 & 0.7 & 0.4 & 0.46 & 0.64 \\
3 & 0.3 & 0.8 & 0.3 & 0.4 & 0.7 \\
2 & 0.2 & 0.9 & 0.2 & 0.34 & 0.76 \\
1 & 0.1 & 1 & 0.2 & 0.36 & 0.84
\end{tabular}

Tab. 3. A counterexample to equation (5). See Example 3.

\subsection{Gradual intervals and fuzzy intervals}

Gradual interval [10, i. e. interval of gradual real numbers, is given by an ordered pair of gradual numbers $\widetilde{X}=\left[\breve{x}^{-}, \breve{x}^{+}\right]$, where $\breve{x}^{-}$is called the left profile (or fuzzy lower bound) of $\widetilde{X}$ and $\breve{x}^{+}$the right profile (or fuzzy upper bound). The set of all gradual intervals will be denoted by $\mathbb{I}(G(\mathbb{R}))$ :

$$
\mathbb{I}(G(\mathbb{R}))=\left\{\left[\breve{x}^{-}, \breve{x}^{+}\right] \mid \breve{x}^{-}, \breve{x}^{+} \in G(\mathbb{R}), \breve{x}^{-} \leq \breve{x}^{+}\right\}
$$

If $\breve{x}^{-}, \breve{x}^{+}$are discrete gradual numbers on the same set $\left\{\alpha_{1}, \ldots, \alpha_{k}\right\}$, then $\left[\breve{x}^{-}, \breve{x}^{+}\right]$is called a discrete gradual interval. The set of all discrete gradual intervals is denoted by $\mathbb{I}\left(D G_{k}(\mathbb{R})\right)$.

A discrete gradual interval $\left[\breve{x}^{-}, \breve{x}^{+}\right]$with strictly increasing left profile $\breve{x}^{-}$and strictly decreasing right profile $\breve{x}^{+}$on $\left\{\alpha_{1}, \ldots, \alpha_{k}\right\}$ characterizes fuzzy interval (fuzzy subset of real line whose $\alpha$-cuts are closed intervals [7, 22]) $f: \mathbb{R} \rightarrow\left\{\alpha_{1}, \ldots, \alpha_{k}\right\}$ in the following way:

$$
f(x)= \begin{cases}\alpha_{i}, & \text { if } x \in\left[\breve{x}^{-}\left(\alpha_{i}\right), \breve{x}^{-}\left(\alpha_{i+1}\right)\right) \cup\left(\breve{x}^{+}\left(\alpha_{i+1}\right), \breve{x}^{+}\left(\alpha_{i}\right)\right], i=1, \ldots, k-1, \\ 1, & \text { if } x \in\left[\breve{x}^{-}(1), \breve{x}^{+}(1)\right] \\ 0, & \text { otherwise. }\end{cases}
$$

Fuzzy intervals $f$ given by (6) will be called fuzzy intervals with finite range to emphasize the difference between them and discrete fuzzy intervals, the latter having discrete domain and the former discrete range. Obviously, $\alpha$-cuts of $f$ in $\alpha_{i}, i=1, \ldots, k$, are closed intervals (see Figure 2):

$$
f_{\alpha_{i}}=\left[\breve{x}^{-}\left(\alpha_{i}\right), \breve{x}^{+}\left(\alpha_{i}\right)\right]
$$

It is worth pointing out that all our results for discrete gradual intervals hold also for fuzzy intervals with finite range. 

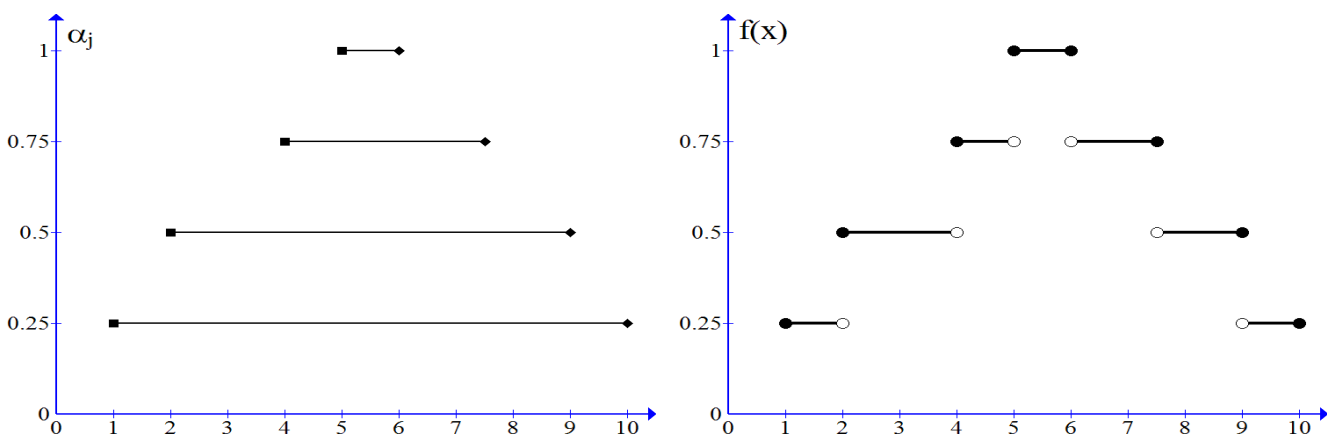

Fig. 2. Discrete gradual interval $\left[\breve{x}^{-}, \breve{x}^{+}\right]$with strictly increasing left profile $\breve{x}^{-}$and strictly decreasing right profile $\breve{x}^{+}$(on the left) and corresponding fuzzy interval $f$ given by (6) (on the right).

We have two natural partial orders on $\mathbb{I}\left(D G_{k}(\mathbb{R})\right)$ based on the partial order $\leq$ and linear order $\preceq_{\mathbb{A}, \mathbf{v}, g}$ on $D G_{k}(\mathbb{R})$ :

$$
\begin{aligned}
{\left[\breve{x}^{-}, \breve{x}^{+}\right] \preceq_{2}\left[\breve{y}^{-}, \breve{y}^{+}\right] \quad \text { iff } \quad\left(\breve{x}^{-} \leq \breve{y}^{-} \quad \text { and } \quad \breve{x}^{+} \leq \breve{y}^{+}\right) } \\
{\left[\breve{x}^{-}, \breve{x}^{+}\right] \preceq_{2}^{\mathbb{A}, \mathbf{v}, g}\left[\breve{y}^{-}, \breve{y}^{+}\right] \quad \text { iff } \quad\left(\breve{x}^{-} \preceq_{\mathbb{A}, \mathbf{v}, g} \breve{y}^{-} \quad \text { and } \quad \breve{x}^{+} \preceq_{\mathbb{A}, \mathbf{v}, g} \breve{y}^{+}\right) }
\end{aligned}
$$

which correspond to commonly used partial order of 'standard' intervals: $[a, b] \leq_{2}[c, d]$ iff $a \leq c, b \leq d$. Clearly, neither $\preceq_{2}$ nor $\preceq_{2}^{\mathbb{A}, \mathbf{v}, g}$ is linear. We emphasize that the order $\preceq_{2}$ coincides (in the sense of the following theorem) with the order $\sqsubseteq_{T 2}$ of membership grades $f, g$ of type- 2 fuzzy sets. Recall that $\sqsubseteq_{T 2}$ is given by:

$$
f \sqsubseteq_{T 2} g \text { iff } f \sqcap g=f, \quad(f \sqcap g)(z)=\sup _{x \wedge y=z}(f(x) \wedge g(y)),
$$

where $f, g$ are functions from $[0,1]$ to $[0,1]$ (called also fuzzy truth values). See [13, 20, 26, 28.

Theorem 3.1. The orders $\preceq_{2}$ and $\sqsubseteq_{T 2}$ coincide on the set $G V \subseteq \mathbb{I}\left(D G_{k}([0,1])\right)$ of all discrete gradual intervals with strictly increasing left profiles and strictly decreasing right profiles, i. e.,

$$
\left[\breve{x}^{-}, \breve{x}^{+}\right] \preceq_{2}\left[\breve{y}^{-}, \breve{y}^{+}\right] \quad \text { iff } \quad f \sqsubseteq_{T 2} g \text {, }
$$

where $\left[\breve{x}^{-}, \breve{x}^{+}\right],\left[\breve{y}^{-}, \breve{y}^{+}\right] \in G V$, and $f, g$ arise by equation (6) from $\left[\breve{x}^{-}, \breve{x}^{+}\right],\left[\breve{y}^{-}, \breve{y}^{+}\right]$, respectively. Moreover,

$$
\left[\breve{x}^{-}, \breve{x}^{+}\right] \preceq_{2}^{\mathbb{A}, \mathbf{v}, g}\left[\breve{y}^{-}, \breve{y}^{+}\right] \quad \text { whenever } \quad f \sqsubseteq T 2 g .
$$

Proof. By [28, Propistition 16] (or [13, Theorem 1]), $f \sqsubseteq_{T 2} g$ holds if and only if $\breve{x}^{-} \leq \breve{y}^{-}$and $\breve{x}^{+} \leq \breve{y}^{+}$, hence if and only if $\left[\breve{x}^{-}, \breve{x}^{+}\right] \preceq_{2}\left[\breve{y}^{-}, \breve{y}^{+}\right]$by (7). Thus (9) is proved. Now let $f \sqsubseteq_{T 2} g$. Then $\left[\breve{x}^{-}, \breve{x}^{+}\right] \preceq_{2}\left[\breve{y}^{-}, \breve{y}^{+}\right]$by $[9]$, hence $\breve{x}^{-} \leq \breve{y}^{-}$and $\breve{x}^{+} \leq \breve{y}^{+}$by (7), consequently, $\breve{x}^{-} \preceq_{\mathbb{A}, \mathbf{v}, g} \breve{y}^{-}$and $\breve{x}^{+} \preceq_{\mathbb{A}, \mathbf{v}, g} \breve{y}^{+}$by (1), and finally, $\left[\breve{x}^{-}, \breve{x}^{+}\right] \preceq_{2}^{\mathbb{A}, \mathbf{v}, g}\left[\breve{y}^{-}, \breve{y}^{+}\right]$by (8). Thus 10 is proved. 


\subsection{Aggregation functions for discrete gradual real numbers}

We generalize definition of aggregation functions on $[0,1]$ (see e. g. [1, 8, 11, 19]) to the gradual numbers setting in a straightforward way.

Definition 3.2. A function $\breve{A}: D G_{k}([0,1])^{n} \rightarrow D G_{k}([0,1])$ is called an $n$-ary aggregation function on $D G_{k}([0,1])$ if it satisfies the conditions:

(A1) $\breve{A}(\breve{0}, \ldots, \breve{0})=\breve{0}$

(A2) $\breve{A}(\breve{1}, \ldots, \breve{1})=\breve{1}$;

(A3) $\breve{x}_{1} \leq \breve{y}_{1}, \ldots, \breve{x}_{n} \leq \breve{y}_{n}$ imply $\breve{A}\left(\breve{x}_{1}, \ldots, \breve{x}_{n}\right) \leq \breve{A}\left(\breve{y}_{1}, \ldots, \breve{y}_{n}\right)$ for all $\breve{x}_{1}, \breve{y}_{1}, \ldots \breve{x}_{n}, \breve{y}_{n} \in$ $D G_{k}([0,1])$.

An $n$-ary aggregation function $\breve{A}$ on $D G_{k}([0,1])$ is said to be induced by $n$-ary aggregation functions $A_{1}, \ldots, A_{k}$ on $[0,1]$ if

$$
\breve{A}\left(\breve{x}_{1}, \ldots, \breve{x}_{n}\right)\left(\alpha_{j}\right)=A_{j}\left(\breve{x}_{1}\left(\alpha_{j}\right), \ldots, \breve{x}_{n}\left(\alpha_{j}\right)\right)
$$

for all $j=1, \ldots, k$. If $A_{1}=A_{2}=\ldots=A_{k}=A, \breve{A}$ is said to be induced by $A$. However, there are aggregation functions on $D G_{k}([0,1])$ which cannot be induced by any aggregation functions $A_{1}, \ldots, A_{k}$, for instance:

$$
\breve{A}\left(\breve{x}_{1}, \breve{x}_{2}\right)\left(\alpha_{j}\right)=\frac{\sum_{i=1}^{k} \breve{x}_{1}\left(\alpha_{i}\right)+\sum_{i=1}^{k} \breve{x}_{2}\left(\alpha_{i}\right)}{2 k} \cdot \breve{x}_{1}\left(\alpha_{j}\right) \breve{x}_{2}\left(\alpha_{j}\right)
$$

for all $j=1, \ldots, k$.

It is easy to check that the DGOWA operator given by Definition 2.10 satisfies (A1) (A3) on $D G_{k}([0,1])$.

Theorem 3.3. DGOWA operator on $D G_{k}([0,1])$ is an aggregation function according to Definition 3.2

Proof. The proof is straightforward and therefore omitted.

\subsection{Linear order of discrete gradual intervals}

Construction of our linear order of gradual intervals generalizes the ideas of [3, 4] where the authors proposed so-called admissible order of intervals.

Definition 3.4. An order $\sqsubseteq$ on $\mathbb{I}\left(D G_{k}(\mathbb{R})\right)$ is called admissible on the set of all discrete gradual intervals, if it is linear and refines the order $\preceq_{2}$ given by (7), i. e., for all $\left[\breve{x}^{-}, \breve{x}^{+}\right],\left[\breve{y}^{-}, \breve{y}^{+}\right] \in \mathbb{I}\left(D G_{k}(\mathbb{R})\right)$,

$$
\left[\breve{x}^{-}, \breve{x}^{+}\right] \sqsubseteq\left[\breve{y}^{-}, \breve{y}^{+}\right] \quad \text { whenever } \quad\left[\breve{x}^{-}, \breve{x}^{+}\right] \preceq_{2}\left[\breve{x}^{-}, \breve{x}^{+}\right] .
$$


Some admissible orders can be generated by a pair of aggregation functions $A, B$ (see [3. Proposition 3.2]). From now on, we only turn our attention to gradual numbers and intervals on $[0,1]$. However, our results remain valid for gradual numbers and intervals on any bounded partially ordered set $(L, \leq)$.

Theorem 3.5. Let $\breve{A}, \breve{B}: D G_{k}([0,1])^{2} \rightarrow D G_{k}([0,1])$ be binary aggregation functions for gradual numbers on $[0,1]$ such that for all $\breve{x}, \breve{y}, \breve{u}, \breve{v} \in D G_{k}([0,1])$ the equalities $\breve{A}(\breve{x}, \breve{y})=\breve{A}(\breve{u}, \breve{v})$ and $\breve{B}(\breve{x}, \breve{y})=\breve{B}(\breve{u}, \breve{v})$ hold if and only if $\breve{x}=\breve{u}$ and $\breve{y}=\breve{v}$. Let $\sqsubseteq_{\breve{A}, \breve{B}}^{\mathbb{A}, \mathbf{v}, g}$ be a relation on $\mathbb{I}\left(D G_{k}([0,1])\right)$ given by:

$$
\begin{aligned}
& {\left[\breve{x}^{-}, \breve{x}^{+}\right] \sqsubseteq_{\breve{A}, \breve{B}}^{\mathbb{A}, \mathbf{v}, g}\left[\breve{y}^{-}, \breve{y}^{+}\right] \quad \quad \breve{A}\left(\breve{x}^{-}, \breve{x}^{+}\right) \prec_{\mathbb{A}, \mathbf{v}, g} \breve{A}\left(\breve{y}^{-}, \breve{y}^{+}\right) \quad \text { or }} \\
& \left(\breve{A}\left(\breve{x}^{-}, \breve{x}^{+}\right)=\breve{A}\left(\breve{y}^{-}, \breve{y}^{+}\right) \text {and } \quad \breve{B}\left(\breve{x}^{-}, \breve{x}^{+}\right) \preceq_{\mathbb{A}, \mathbf{v}, g} \breve{B}\left(\breve{y}^{-}, \breve{y}^{+}\right)\right) \text {. }
\end{aligned}
$$

Then $\sqsubseteq_{\breve{A}, \breve{B}}^{\mathbb{A}, \mathbf{v}, g}$ is an admissible order on $\mathbb{I}\left(D G_{k}([0,1])\right)$.

Proof. Let us first show that $\sqsubseteq_{\breve{A}, \breve{B}}^{\mathbb{A}, \mathbf{v}}$ is a partial order. Reflexivity is obvious. Antisymmetry: Let $\left[\breve{x}^{-}, \breve{x}^{+}\right] \sqsubseteq_{\breve{A}, \breve{B}}^{\mathbb{A}, \mathbf{v}, g}\left[\breve{y}^{-}, \breve{y}^{+}\right]$and $\left[\breve{y}^{-}, \breve{y}^{+}\right] \sqsubseteq_{\breve{A}, \breve{B}}^{\mathbb{A}, \mathbf{v}, g}\left[\breve{x}^{-}, \breve{x}^{+}\right]$. Since $\preceq_{\mathbb{A}, \mathbf{v}, g}$ is linear, it follows that

$$
\breve{A}\left(\breve{x}^{-}, \breve{x}^{+}\right)=\breve{A}\left(\breve{y}^{-}, \breve{y}^{+}\right), \breve{B}\left(\breve{x}^{-}, \breve{x}^{+}\right) \preceq_{\mathbb{A}, \mathbf{v}, g} \breve{B}\left(\breve{y}^{-}, \breve{y}^{+}\right), \breve{B}\left(\breve{y}^{-}, \breve{y}^{+}\right) \preceq_{\mathbb{A}, \mathbf{v}, g} \breve{B}\left(\breve{x}^{-}, \breve{x}^{+}\right),
$$

hence

$$
\breve{A}\left(\breve{x}^{-}, \breve{x}^{+}\right)=\breve{A}\left(\breve{y}^{-}, \breve{y}^{+}\right), \breve{B}\left(\breve{x}^{-}, \breve{x}^{+}\right)=\breve{B}\left(\breve{y}^{-}, \breve{y}^{+}\right)
$$

and consequently, by the assumption of theorem, $\breve{x}^{-}=\breve{y}^{-}$and $\breve{x}^{+}=\breve{y}^{+}$.

Transitivity: assumption $\left[\breve{x}^{-}, \breve{x}^{+}\right] \sqsubseteq_{\breve{A}, \breve{B}}^{\mathbb{A}, \mathbf{v}, g}\left[\breve{y}^{-}, \breve{y}^{+}\right]$and $\left[\breve{y}^{-}, \breve{y}^{+}\right] \sqsubseteq_{\breve{A}, \breve{B}}^{\mathbb{A}, \mathbf{v}, g}\left[\breve{z}^{-}, \breve{z}^{+}\right]$leads to one of the following four cases:

1. $\breve{A}\left(\breve{x}^{-}, \breve{x}^{+}\right) \prec_{\mathbb{A}, \mathbf{v}, g} \breve{A}\left(\breve{y}^{-}, \breve{y}^{+}\right)$and $\breve{A}\left(\breve{y}^{-}, \breve{y}^{+}\right) \prec_{\mathbb{A}, \mathbf{v}, g} \breve{A}\left(\breve{z}^{-}, \breve{z}^{+}\right)$, which implies $\breve{A}\left(\breve{x}^{-}, \breve{x}^{+}\right) \prec_{\mathbb{A}, \mathbf{v}, g} \breve{A}\left(\breve{z}^{-}, \breve{z}^{+}\right)$.

2. $\breve{A}\left(\breve{x}^{-}, \breve{x}^{+}\right)=\breve{A}\left(\breve{y}^{-}, \breve{y}^{+}\right), \breve{B}\left(\breve{x}^{-}, \breve{x}^{+}\right) \preceq_{\mathbb{A}, \mathbf{v}, g} \breve{B}\left(\breve{y}^{-}, \breve{y}^{+}\right), \breve{A}\left(\breve{y}^{-}, \breve{y}^{+}\right)=\breve{A}\left(\breve{z}^{-}, \breve{z}^{+}\right)$and $\breve{B}\left(\breve{y}^{-}, \breve{y}^{+}\right) \preceq_{\mathbb{A}, \mathbf{v}, g} \breve{B}\left(\breve{z}^{-}, \breve{z}^{+}\right)$, which gives $\breve{A}\left(\breve{x}^{-}, \breve{x}^{+}\right)=\breve{A}\left(\breve{z}^{-}, \breve{z}^{+}\right)$and $\breve{B}\left(\breve{x}^{-}, \breve{x}^{+}\right) \preceq_{\mathbb{A}, \mathbf{v}, g}$ $\breve{B}\left(\breve{z}^{-}, \breve{z}^{+}\right)$.

3. $\breve{A}\left(\breve{x}^{-}, \breve{x}^{+}\right) \prec_{\mathbb{A}, \mathbf{v}, g} \breve{A}\left(\breve{y}^{-}, \breve{y}^{+}\right), \breve{A}\left(\breve{y}^{-}, \breve{y}^{+}\right)=\breve{A}\left(\breve{z}^{-}, \breve{z}^{+}\right)$and $\breve{B}\left(\breve{y}^{-}, \breve{y}^{+}\right) \preceq_{\mathbb{A}, \mathbf{v}, g} \breve{B}\left(\breve{z}^{-}, \breve{z}^{+}\right)$, which leads to $\breve{A}\left(\breve{x}^{-}, \breve{x}^{+}\right) \prec_{\mathbb{A}, \mathbf{v}, g} \breve{A}\left(\breve{z}^{-}, \breve{z}^{+}\right)$.

4. $\breve{A}\left(\breve{x}^{-}, \breve{x}^{+}\right)=\breve{A}\left(\breve{y}^{-}, \breve{y}^{+}\right), \breve{B}\left(\breve{x}^{-}, \breve{x}^{+}\right) \preceq_{\mathbb{A}, \mathbf{v}, g} \breve{B}\left(\breve{y}^{-}, \breve{y}^{+}\right)$and $\breve{A}\left(\breve{y}^{-}, \breve{y}^{+}\right) \prec_{\mathbb{A}, \mathbf{v}, g} \breve{A}\left(\breve{z}^{-}, \breve{z}^{+}\right)$, which implies $\breve{A}\left(\breve{x}^{-}, \breve{x}^{+}\right) \prec_{\mathbb{A}, \mathbf{v}, g} \breve{A}\left(\breve{z}^{-}, \breve{z}^{+}\right)$.

From each of the four cases it follows that $\left[\breve{x}^{-}, \breve{x}^{+}\right] \sqsubseteq \breve{A}, \breve{B}\left[\breve{z}^{-}, \breve{z}^{+}\right]$, thus $\sqsubseteq_{\breve{A}, \breve{B}}^{\mathbb{A}, \mathbf{v}, g}$ is a partial order. Linearity immediately follows from 11 and linearity of $\preceq_{\mathbb{A}, \mathbf{v}, g}$.

Aggregation functions $\breve{A}, \breve{B}$ for discrete gradual numbers which satisfy the assumption of Theorem 3.5 can be obtained by aggregation functions for real numbers with similar property. 
Lemma 3.6. Let $\breve{A}, \breve{B}: D G_{k}([0,1])^{2} \rightarrow D G_{k}([0,1])$ be binary aggregation functions for gradual numbers on $[0,1]$ induced by binary aggregation functions $A, B:[0,1]^{2} \rightarrow[0,1]$, respectively. Let for all $x, y, u, v \in[0,1]$ the equalities $A(x, y)=A(u, v)$ and $B(x, y)=$ $B(u, v)$ hold if and only if $x=u$ and $y=v$. Then for all $\breve{x}, \breve{y}, \breve{u}, \breve{v} \in D G_{k}([0,1])$ the equalities $\breve{A}(\breve{x}, \breve{y})=\breve{A}(\breve{u}, \breve{v})$ and $\breve{B}(\breve{x}, \breve{y})=\breve{B}(\breve{u}, \breve{v})$ hold if and only if $\breve{x}=\breve{u}$ and $\breve{y}=\breve{v}$.

Proof. The proof is straightforward and therefore omitted.

From an admissible order of discrete gradual intervals one can induce a linear order of fuzzy intervals (of a special kind).

Corollary 3.7. Let $V$ be the set of all fuzzy intervals on $[0,1]$ with finite range $f$ : $[0,1] \rightarrow\left\{\alpha_{1}, \ldots, \alpha_{k}\right\}$, where $0<\alpha_{1}<\ldots<\alpha_{k}=1$, whose $\alpha$-cuts are nested closed intervals $f_{\alpha_{i}}=\left[\underline{f_{\alpha_{i}}}, \overline{f_{\alpha_{i}}}\right]$ for all $i=1, \ldots, k$. Then the following relation $\sqsubseteq_{V}$ on $V$ is a linear order:

$$
f \sqsubseteq V h \quad \text { iff } \quad\left[\breve{x}^{-}, \breve{x}^{+}\right] \sqsubseteq_{\breve{A}, \breve{B}}^{\mathbb{A}, \mathbf{v}, g}\left[\breve{y}^{-}, \breve{y}^{+}\right]
$$

where $\breve{x}^{-}\left(\alpha_{i}\right)=\underline{f_{\alpha_{i}}}, \breve{x}^{+}\left(\alpha_{i}\right)=\overline{f_{\alpha_{i}}}, \breve{y}^{-}\left(\alpha_{i}\right)=\underline{h_{\alpha_{i}}}, \breve{y}^{+}\left(\alpha_{i}\right)=\overline{h_{\alpha_{i}}}$, and $\sqsubseteq_{\breve{A}, \breve{B}, g}^{\mathbb{A}, \mathbf{s}}$ is an admissible order given in Theorem 3.5

Proof. The proof is based on the observation that equation (6) defines a bijection from the set of all discrete gradual intervals with strictly increasing left profiles and strictly decreasing right profiles to the set $V$.

Example 4. Let extended aggregation function $\mathbb{A}_{\mathbf{v}}$ be weighted average. Consider the discrete gradual intervals $\widetilde{X}=\left[\breve{x}^{-}, \breve{x}^{+}\right], \widetilde{Y}=\left[\breve{y}^{-}, \breve{y}^{+}\right]$and $\widetilde{Z}=\left[\breve{z}^{-}, \breve{z}^{+}\right]$given by Table 4 . Let an aggregation function $\breve{A}$ be induced by arithmetic mean $A(a, b)=\frac{a+b}{2}$ and $\breve{B}$ be induced by geometric mean $B(a, b)=\sqrt{a b}$. Then, for weighting vector $\mathbf{v}=(0.1, \ldots, 0.1)$, WAs are:

$$
\begin{aligned}
& \sum_{j=1}^{10} v_{j} \breve{A}\left(\breve{x}^{-}, \breve{x}^{+}\right)\left(\alpha_{j}\right)=\sum_{j=1}^{10} v_{j} \breve{A}\left(\breve{y}^{-}, \breve{y}^{+}\right)\left(\alpha_{j}\right)=0.385 \\
& \sum_{j=1}^{10} v_{j} \breve{A}\left(\breve{z}^{-}, \breve{z}^{+}\right)\left(\alpha_{j}\right)=0.975
\end{aligned}
$$

which means that $\widetilde{X}, \widetilde{Y} \sqsubseteq_{\breve{A}, \breve{B}}^{\mathbb{A}, \mathbf{v}, g} \widetilde{Z}$. Since $\breve{A}\left(\breve{x}^{-}, \breve{x}^{+}\right)=\breve{A}\left(\breve{y}^{-}, \breve{y}^{+}\right)$, we apply $\breve{B}$ to compare $\tilde{X}$ and $\tilde{Y}$. From

$$
\sum_{j=1}^{10} v_{j} \breve{B}\left(\breve{x}^{-}, \breve{x}^{+}\right)\left(\alpha_{j}\right)=0.351, \quad \sum_{j=1}^{10} v_{j} \breve{B}\left(\breve{y}^{-}, \breve{y}^{+}\right)\left(\alpha_{j}\right)=0.331
$$

it follows that $\tilde{Y} \sqsubseteq_{\breve{A}, \breve{B}}^{\mathbb{A}, \mathbf{v} g} \widetilde{X} \sqsubseteq_{\breve{A}, \breve{B}}^{\mathbb{A}, \mathbf{v}, g} \widetilde{Z}$. 
Now we will consider the same situation but use another weighting vector $\mathbf{u}=$ $(0.04,0.06,0.08,0.1,0.1,0.1,0.1,0.12,0.14,0.16)$. Then

$$
\begin{aligned}
& \sum_{j=1}^{10} u_{j} \breve{A}\left(\breve{x}^{-}, \breve{x}^{+}\right)\left(\alpha_{j}\right)=0.335, \quad \sum_{j=1}^{10} u_{j} \breve{A}\left(\breve{y}^{-}, \breve{y}^{+}\right)\left(\alpha_{j}\right)=0.34 \\
& \sum_{j=1}^{10} u_{j} \breve{A}\left(\breve{z}^{-}, \breve{z}^{+}\right)\left(\alpha_{j}\right)=0.973
\end{aligned}
$$

which implies $\tilde{X} \sqsubseteq_{\breve{A}, \breve{B}}^{\mathbb{A}, \mathbf{u}, g} \widetilde{Y} \sqsubseteq_{\breve{A}, \breve{B}}^{\mathbb{A}, \mathbf{u}, g} \widetilde{Z}$.

We can see that we obtained different order for different weighting vectors. Recall that, in this example, all the results hold for any redistribution function $g$ because we did not need to redistribute the weights.

\begin{tabular}{c|ccc|ccc|cc}
$\alpha_{j}$ & $\widetilde{X}$ & $\tilde{Y}$ & $\widetilde{Z}$ & $\breve{A}\left(\breve{x}^{-}, \breve{x}^{+}\right)$ & $\breve{A}\left(\breve{y}^{-}, \breve{y}^{+}\right)$ & $\breve{A}\left(\breve{z}^{-}, \breve{z}^{+}\right)$ & $\breve{B}\left(\breve{x}^{-}, \breve{x}^{+}\right)$ & $\breve{B}\left(\breve{y}^{-}, \breve{y}^{+}\right)$ \\
\hline 1 & {$[0.1,0.6]$} & {$[0.3,0.4]$} & {$[1,1]$} & 0.35 & 0.35 & 1 & 0.245 & 0.346 \\
0.9 & {$[0.1,0.6]$} & {$[0.3,0.4]$} & {$[1,1]$} & 0.35 & 0.35 & 1 & 0.245 & 0.346 \\
0.8 & {$[0.2,0.6]$} & {$[0.3,0.5]$} & {$[0.9,1]$} & 0.4 & 0.4 & 0.95 & 0.346 & 0.387 \\
0.7 & {$[0.2,0.5]$} & {$[0.2,0.5]$} & {$[0.9,1]$} & 0.35 & 0.35 & 0.95 & 0.316 & 0.316 \\
0.6 & {$[0.3,0.5]$} & {$[0.2,0.6]$} & {$[0.9,1]$} & 0.4 & 0.4 & 0.95 & 0.387 & 0.346 \\
0.5 & {$[0.3,0.5]$} & {$[0.2,0.6]$} & {$[0.9,1]$} & 0.4 & 0.4 & 0.95 & 0.387 & 0.346 \\
0.4 & {$[0.3,0.5]$} & {$[0.2,0.6]$} & {$[0.9,1]$} & 0.4 & 0.4 & 0.95 & 0.387 & 0.346 \\
0.3 & {$[0.4,0.4]$} & {$[0.2,0.6]$} & {$[1,1]$} & 0.4 & 0.4 & 1 & 0.4 & 0.346 \\
0.2 & {$[0.4,0.4]$} & {$[0.1,0.7]$} & {$[1,1]$} & 0.4 & 0.4 & 1 & 0.4 & 0.265 \\
0.1 & {$[0.4,0.4]$} & {$[0.1,0.7]$} & {$[1,1]$} & 0.4 & 0.4 & 1 & 0.4 & 0.265 \\
\hline$W A_{v}$ & & & & 0.385 & 0.385 & 0.975 & 0.351 & 0.331 \\
$W A_{u}$ & & & & 0.335 & 0.34 & 0.973 & &
\end{tabular}

Tab. 4. The discrete gradual intervals $\widetilde{X}, \widetilde{Y}, \widetilde{Z}$ from Example 4 .

Aggregation function $\breve{A}$ is induced by arithmetic mean, $\breve{B}$ is induced by geometric mean, $W A_{v}$ denotes the weighted average associated with weighting vector $\mathbf{v}$ and similarly for $W A_{u}$ and $\mathbf{u}$.

\subsection{OWA operators for discrete gradual intervals}

Since we proposed a linear order of discrete gradual intervals, we can define OWA operator for these objects.

Definition 3.8. Let $\breve{\mathbf{w}}=\left(\breve{w}_{1}, \ldots, \breve{w}_{n}\right) \in D G_{k}([0,1])^{n}$ with $\breve{w}_{1}+\ldots+\breve{w}_{n}=\breve{1}$ be a weighting vector of discrete gradual numbers. A discrete gradual intervals OWA operator (DGIOWA for short) associated with $\breve{\mathbf{w}}$ is a mapping $D G I O W A_{\breve{\mathbf{w}}}^{\sqsubseteq}: \mathbb{I}\left(D G_{k}([0,1])\right)^{n} \rightarrow$ $\mathbb{I}\left(D G_{k}([0,1])\right)$ defined by

$$
\operatorname{DGIOW} A_{\widetilde{\mathbf{w}}}^{\sqsubseteq}\left(\left[\breve{x}_{1}^{-}, \breve{x}_{1}^{+}\right], \ldots,\left[\breve{x}_{n}^{-}, \breve{x}_{n}^{+}\right]\right)=\sum_{i=1}^{n} \breve{w}_{i} \cdot\left[\breve{x}_{(i)}^{-}, \breve{x}_{(i)}^{+}\right],
$$


where $\left[\breve{x}_{(i)}^{-}, \breve{x}_{(i)}^{+}\right], i=1, \ldots, n$, denote the $i$ th greatest component of the input vector $\left(\left[\breve{x}_{1}^{-}, \breve{x}_{1}^{+}\right], \ldots,\left[\breve{x}_{n}^{-}, \breve{x}_{n}^{+}\right]\right)$with respect to an admissible order $\sqsubseteq$.

Application of the interval [21] and gradual number [10] arithmetic to equation (12) leads to computation of $k$ independent results:

$$
\operatorname{DGIOW} A_{\breve{\mathbf{w}}}^{\sqsubseteq}\left(\left[\breve{x}_{1}^{-}, \breve{x}_{1}^{+}\right], \ldots,\left[\breve{x}_{n}^{-}, \breve{x}_{n}^{+}\right]\right)\left(\alpha_{j}\right)=\sum_{i=1}^{n}\left[\breve{w}_{i}\left(\alpha_{j}\right) \cdot \breve{x}_{(i)}^{-}\left(\alpha_{j}\right), \breve{w}_{i}\left(\alpha_{j}\right) \breve{x}_{(i)}^{+}\left(\alpha_{j}\right)\right]
$$

for all $j=1, \ldots, k$. We emphasize that $D G I O W A_{\breve{\mathbf{w}}}^{\sqsubseteq}$ is very easily computable by 13 .

The DGIOWA operator satisfies basic properties of aggregation functions, i. e. the boundary conditions and monotonicity (with respect to partial order $\preceq_{2}$ ).

Theorem 3.9. For any operator $D G I O W A_{\breve{\mathbf{w}}}^{\sqsubset}$ on $\mathbb{I}\left(D G_{k}([0,1])\right)$ it holds:

- $D G I O W A_{\breve{\mathbf{w}}}^{\sqsubseteq}([\breve{0}, \breve{0}], \ldots,[\breve{0}, \breve{0}])=[\breve{0}, \breve{0}]$;

- $D G I O W A_{\breve{\mathbf{w}}}^{\sqsubseteq}([\breve{1}, \breve{1}], \ldots,[\breve{1}, \breve{1}])=[\breve{1}, \breve{1}]$;

- $\left[\breve{x}_{1}^{-}, \breve{x}_{1}^{+}\right] \preceq_{2}\left[\breve{y}_{1}^{-}, \breve{y}_{1}^{+}\right], \ldots,\left[\breve{x}_{n}^{-}, \breve{x}_{n}^{+}\right] \preceq_{2}\left[\breve{y}_{n}^{-}, \breve{y}_{n}^{+}\right]$imply $D G I O W A_{\breve{\mathbf{w}}}^{\sqsubset}\left(\left[\breve{x}_{1}^{-}, \breve{x}_{1}^{+}\right], \ldots,\left[\breve{x}_{n}^{-}, \breve{x}_{n}^{+}\right]\right) \preceq_{2} D G I O W A_{\breve{\mathbf{w}}}^{\sqsubseteq}\left(\left[\breve{y}_{1}^{-}, \breve{y}_{1}^{+}\right], \ldots,\left[\breve{y}_{n}^{-}, \breve{y}_{n}^{+}\right]\right)$for all $\left[\breve{x}_{1}^{-}, \breve{x}_{1}^{+}\right],\left[\breve{y}_{1}^{-}, \breve{y}_{1}^{+}\right], \ldots,\left[\breve{x}_{n}^{-}, \breve{x}_{n}^{+}\right],\left[\breve{y}_{n}^{-}, \breve{y}_{n}^{+}\right] \in \mathbb{I}\left(D G_{k}([0,1])\right)$.

Proof. The proof immediately follows from Definition 3.8 and equations $(13),(7)$.

The important point to note here is that the third property of the previous theorem, which holds for the partial order $\preceq_{2}$, does not hold for admissible orders $\sqsubseteq$ in general. For admissible orders it only holds if the weights are real numbers, i. e., $\breve{\mathbf{w}}=\left(\breve{w}_{1}, \ldots, \breve{w}_{n}\right)$ with $\breve{w}_{i} \stackrel{\circ}{=} w_{i} \in \mathbb{R}, i=1, \ldots, n$.

Note that, for some appropriate orders of intervals and gradual intervals, DGIOWA operator is an extension of $I V O W A$, which is an operator on the set $\mathbb{I}([0,1])$ of all closed intervals of real numbers proposed in [4], and also of 'standard' OWA operator for real numbers.

Theorem 3.10. Let $\preceq_{A, B}$ be an admissible order on $\mathbb{I}([0,1])$ generated by $A, B$, and let $\sqsubseteq_{\breve{A}, \breve{B}}^{\mathbb{A}, \mathbf{v}, g}$ be an admissible order on $\mathbb{I}\left(D G_{k}([0,1])\right)$ generated by $\breve{A}, \breve{B}$, where $\breve{A}$ is induced by $A$ and $\breve{B}$ is induced by $B$. Let $\mathbf{w}=\left(w_{1}, \ldots, w_{n}\right) \in[0,1]^{n}$ with $w_{1}+\ldots+w_{n}=1$, and let $\breve{\mathbf{w}}=\left(\breve{w}_{1}, \ldots, \breve{w}_{n}\right)$ with $\breve{w}_{i} \stackrel{\circ}{=} w_{i}$ for all $i=1, \ldots, n$. Then

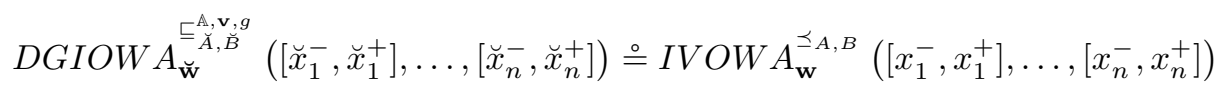

where $\breve{x}_{i}^{-} \stackrel{\circ}{=} x_{i}^{-}$and $\breve{x}_{i}^{+} \stackrel{\circ}{=} x_{i}^{+}$for all $i=1, \ldots, n$. Moreover, for each admissible order $\sqsubseteq$ on $\mathbb{I}\left(D G_{k}([0,1])\right)$ it holds

$$
D G I O W A_{\breve{\mathbf{w}}}^{\sqsubseteq}\left(\left[\breve{x}_{1}, \breve{x}_{1}\right], \ldots,\left[\breve{x}_{n}, \breve{x}_{n}\right]\right) \stackrel{\circ}{=} W A_{\mathbf{w}}\left(x_{1}, \ldots, x_{n}\right)
$$

where $\breve{x}_{i} \stackrel{\circ}{=} x_{i}$ for all $i=1, \ldots, n$. 
Proof. 1. First observe that, for all $i, j=1, \ldots, k, j \neq k,\left[x_{i}^{-}, x_{i}^{+}\right] \preceq_{A, B}\left[x_{j}^{-}, x_{j}^{+}\right]$ implies

$$
\begin{gathered}
A\left(x_{i}^{-}, x_{i}^{+}\right)<A\left(x_{j}^{-}, x_{j}^{+}\right) \text {or } \\
\left(A\left(x_{i}^{-}, x_{i}^{+}\right)=A\left(x_{j}^{-}, x_{j}^{+}\right) \text {and } B\left(x_{i}^{-}, x_{i}^{+}\right) \leq B\left(x_{j}^{-}, x_{j}^{+}\right)\right),
\end{gathered}
$$

hence

$$
\begin{gathered}
\breve{A}\left(\breve{x}_{i}^{-}, \breve{x}_{i}^{+}\right) \prec_{\mathbb{A}, \mathbf{v}, g} \breve{A}\left(\breve{x}_{j}^{-}, \breve{x}_{j}^{+}\right) \text {or } \\
\left(\breve{A}\left(\breve{x}_{i}^{-}, \breve{x}_{i}^{+}\right)=\breve{A}\left(\breve{x}_{j}^{-}, \breve{x}_{j}^{+}\right) \text {and } \breve{B}\left(\breve{x}_{i}^{-}, \breve{x}_{i}^{+}\right) \preceq_{\mathbb{A}, \mathbf{v}, g} \breve{B}\left(\breve{x}_{j}^{-}, \breve{x}_{j}^{+}\right),\right.
\end{gathered}
$$

thus $\left[\breve{x}_{i}^{-}, \breve{x}_{i}^{+}\right] \sqsubseteq_{\breve{A}, \breve{B}}^{\mathbb{A}, \mathbf{v}, g}\left[\breve{x}_{j}^{-}, \breve{x}_{j}^{+}\right]$.

Therefore, by linearity of $\preceq_{A, B}$ and $\sqsubseteq_{\breve{A}, \breve{B}}^{\mathbb{A}, \mathbf{v}, g}$,

$$
\left[\breve{x}_{(n)}^{-}, \breve{x}_{(n)}^{+}\right] \sqsubseteq_{\breve{A}, \breve{B}}^{\mathbb{A}, \mathbf{v}, g} \ldots \sqsubseteq_{\breve{A}, \breve{B}}^{\mathbb{A}, \mathbf{v}, g}\left[\breve{x}_{(1)}^{-}, \breve{x}_{(1)}^{+}\right] \quad \Leftrightarrow \quad\left[x_{(n)}^{-}, x_{(n)}^{+}\right] \preceq_{A, B} \ldots \preceq_{A, B}\left[x_{(1)}^{-}, x_{(1)}^{+}\right],
$$

and finally,

$$
\begin{aligned}
& \operatorname{DGIOW} A_{\breve{\mathbf{w}}}^{\stackrel{\check{A}^{\mathrm{A}, \mathbf{v}, g}, \breve{B}}{(}}\left(\left[\breve{x}_{1}^{-}, \breve{x}_{1}^{+}\right], \ldots,\left[\breve{x}_{n}^{-}, \breve{x}_{n}^{+}\right]\right)=\sum_{i=1}^{n} \breve{w}_{i} \cdot\left[\breve{x}_{(i)}^{-}, \breve{x}_{(i)}^{+}\right] \stackrel{\circ}{=} \\
& \stackrel{\circ}{=} \sum_{i=1}^{n} w_{i} \cdot\left[x_{(i)}^{-}, x_{(i)}^{+}\right]=I V O W A_{\mathbf{w}}^{\preceq_{A, B}}\left(\left[x_{1}^{-}, x_{1}^{+}\right], \ldots,\left[x_{n}^{-}, x_{n}^{+}\right]\right) .
\end{aligned}
$$

2. Observe that $x_{(n)} \leq \ldots \leq x_{(1)}$ implies $\breve{x}_{(n)} \leq \ldots \leq \breve{x}_{(1)}$, hence $\left[\breve{x}_{(n)}, \breve{x}_{(n)}\right] \preceq_{2}$ $\ldots \preceq_{2}\left[\breve{x}_{(1)}, \breve{x}_{(1)}\right]$, and consequently $\left[\breve{x}_{(n)}, \breve{x}_{(n)}\right] \sqsubseteq \ldots \sqsubseteq\left[\breve{x}_{(1)}, \breve{x}_{(1)}\right]$.

Finally

$$
\begin{aligned}
& \operatorname{DGIOW} A_{\breve{\mathbf{w}}}^{\sqsubseteq}\left(\left[\breve{x}_{1}, \breve{x}_{1}\right], \ldots,\left[\breve{x}_{n}, \breve{x}_{n}\right]\right)=\sum_{i=1}^{n} \breve{w}_{i} \cdot\left[\breve{x}_{(i)}, \breve{x}_{(i)}\right] \stackrel{\circ}{=} \\
& \stackrel{\circ}{=} \sum_{i=1}^{n} w_{i} \cdot\left[x_{(i)}, x_{(i)}\right]=\sum_{i=1}^{n} w_{i} \cdot x_{(i)}=O W A_{\mathbf{w}}\left(x_{1}, \ldots, x_{n}\right) .
\end{aligned}
$$

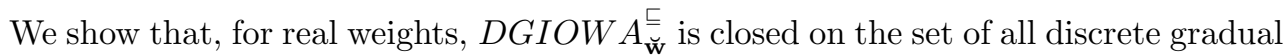
intervals with strictly increasing left profiles and strictly decreasing right profiles, which means that, according to $(6), D G I O W A_{\breve{\mathbf{w}}}^{\sqsubset}$ is closed on the set $V$ of all fuzzy intervals with finite range.

Theorem 3.11. Let $G V \subseteq \mathbb{I}\left(D G_{k}([0,1])\right)$ be the set of all discrete gradual intervals with strictly increasing left profiles and strictly decreasing right profiles. If

$$
\left[\breve{x}_{1}^{-}, \breve{x}_{1}^{+}\right], \ldots,\left[\breve{x}_{n}^{-}, \breve{x}_{n}^{+}\right] \in G V,
$$

then

$$
\operatorname{DGIOW} A_{\breve{\mathbf{w}}}^{\sqsubseteq}\left(\left[\breve{x}_{1}^{-}, \breve{x}_{1}^{+}\right], \ldots,\left[\breve{x}_{n}^{-}, \breve{x}_{n}^{+}\right]\right) \in G V
$$

where $\breve{\mathbf{w}}=\left(\breve{w}_{1}, \ldots, \breve{w}_{n}\right)$ with $\breve{w}_{i} \stackrel{\circ}{=} w_{i}, i=1, \ldots, n$, for some weighting vector of real numbers $\mathbf{w}=\left(w_{1}, \ldots, w_{n}\right)$. 
Proof. For all $j=1, \ldots, k-1, i=1, \ldots, n$ it holds: $\breve{w}_{i}\left(\alpha_{j}\right)=\breve{w}_{i}\left(\alpha_{j+1}\right), \breve{x}_{i}^{-}\left(\alpha_{j}\right)<$ $\breve{x}_{i}^{-}\left(\alpha_{j+1}\right), \breve{x}_{i}^{+}\left(\alpha_{j}\right)>\breve{x}_{i}^{+}\left(\alpha_{j+1}\right)$ and by eq. $\sqrt{13} D G I O W A_{\breve{\mathbf{w}}}^{\sqsubseteq}\left(\left[\breve{x}_{1}^{-}, \breve{x}_{1}^{+}\right], \ldots,\left[\breve{x}_{n}^{-}, \breve{x}_{n}^{+}\right]\right) \in$ $G V$.

Example 5. Consider $\widetilde{Y}_{1}, \widetilde{Y}_{2}, \widetilde{Y}_{3}$ and $\mathbb{A}, \breve{A}, \breve{B}, \mathbf{v}, \mathbf{u}$ from Example 4. Let $\breve{\mathbf{w}}=\left(\breve{w}_{1}, \breve{w}_{2}, \breve{w}_{3}\right)$ be given by Table 5 . Then, by Example 4, $\widetilde{Y}_{2} \sqsubseteq_{\breve{A}, \breve{B}}^{\mathbb{A}, \mathbf{v}, g} \widetilde{Y}_{1} \sqsubseteq_{\breve{A}, \breve{B}}^{\mathbb{A}, \mathbf{v}, g} \tilde{Y}_{3}$. The resulting

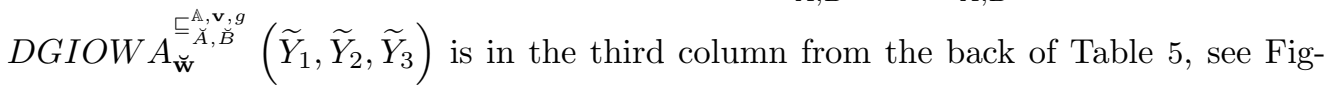
ure 3. However, when we consider weighting vector $\mathbf{u}$ instead of $\mathbf{v}$, we obtain different

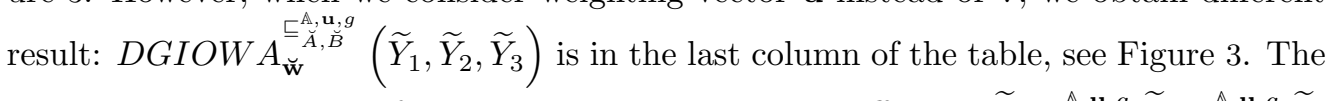
reason is that the order of discrete gradual intervals is different: $\widetilde{Y}_{1} \sqsubseteq_{\breve{A}, \breve{B}}^{\mathbb{A}, \mathbf{u}, g} \widetilde{Y}_{2} \coprod_{\breve{A}, \breve{B}}^{\mathbb{A}, \mathbf{u}, g} \widetilde{Y}_{3}$ (see Example 4).

\begin{tabular}{c|ccc|ccc|cc|cc}
$\alpha_{j}$ & $\widetilde{Y}_{1}$ & $\widetilde{Y}_{2}$ & $\widetilde{Y}_{3}$ & $\breve{w}_{1}$ & $\breve{w}_{2}$ & $\breve{w}_{3}$ & $\mathbf{v}$ & $D G I O W A_{\mathbf{v}}$ & $\mathbf{u}$ & DGIOWA \\
\hline 1 & {$[0.1,0.6]$} & {$[0.3,0.4]$} & {$[1,1]$} & 0.1 & 0.3 & 0.6 & 0.1 & {$[0.31,0.52]$} & 0.16 & {$[0.25,0.58]$} \\
0.9 & {$[0.1,0.6]$} & {$[0.3,0.4]$} & {$[1,1]$} & 0.2 & 0.2 & 0.6 & 0.1 & {$[0.4,0.56]$} & 0.14 & {$[0.32,0.64]$} \\
0.8 & {$[0.2,0.6]$} & {$[0.3,0.5]$} & {$[0.9,1]$} & 0.3 & 0.2 & 0.5 & 0.1 & {$[0.46,0.67]$} & 0.12 & {$[0.43,0.7]$} \\
0.7 & {$[0.2,0.5]$} & {$[0.2,0.5]$} & {$[0.9,1]$} & 0.4 & 0.1 & 0.5 & 0.1 & {$[0.48,0.7]$} & 0.1 & {$[0.48,0.7]$} \\
0.6 & {$[0.3,0.5]$} & {$[0.2,0.6]$} & {$[0.9,1]$} & 0.4 & 0.1 & 0.5 & 0.1 & {$[0.49,0.75]$} & 0.1 & {$[0.53,0.71]$} \\
0.5 & {$[0.3,0.5]$} & {$[0.2,0.6]$} & {$[0.9,1]$} & 0.4 & 0.1 & 0.5 & 0.1 & {$[0.49,0.75]$} & 0.1 & {$[0.53,0.71]$} \\
0.4 & {$[0.3,0.5]$} & {$[0.2,0.6]$} & {$[0.9,1]$} & 0.3 & 0.1 & 0.6 & 0.1 & {$[0.42,0.71]$} & 0.1 & {$[0.47,0.66]$} \\
0.3 & {$[0.4,0.4]$} & {$[0.2,0.6]$} & {$[1,1]$} & 0.3 & 0.1 & 0.6 & 0.1 & {$[0.46,0.7]$} & 0.08 & {$[0.56,0.6]$} \\
0.2 & {$[0.4,0.4]$} & {$[0.1,0.7]$} & {$[1,1]$} & 0.2 & 0.1 & 0.7 & 0.1 & {$[0.31,0.73]$} & 0.06 & {$[0.49,0.55]$} \\
0.1 & {$[0.4,0.4]$} & {$[0.1,0.7]$} & {$[1,1]$} & 0.1 & 0.1 & 0.8 & 0.1 & {$[0.22,0.7]$} & 0.04 & {$[0.43,0.49]$}
\end{tabular}

\footnotetext{
Tab. 5. Comparison of DGIOW $A_{\breve{\mathbf{w}}}^{\complement_{A}^{\mathrm{A}, \mathbf{v}^{,}, g}}\left(\widetilde{Y}_{1}, \widetilde{Y}_{2}, \widetilde{Y}_{3}\right)$ and

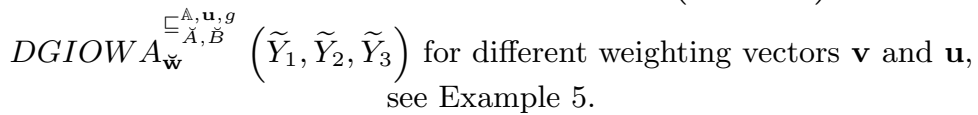

\section{APPLICATION TO MULTI-EXPERT DECISION MAKING}

In this section a new procedure of solving a multi-expert decision making (MEDM) problem using discrete gradual intervals is presented. As can be seen from given example, an assessment of particular criterion in form of a gradual interval which is not a fuzzy set may arise in a natural way. Thus, the problem cannot be solved by existing methods based on aggregation of fuzzy sets.

A decision maker $(\mathrm{DM})$ considers one of the $n$ alternatives alt $_{1}, \ldots$, alt $_{n}$ according to the $m$ criterions $c_{1}, \ldots, c_{m}$. We propose the following algorithm to solve this MEDM problem: 

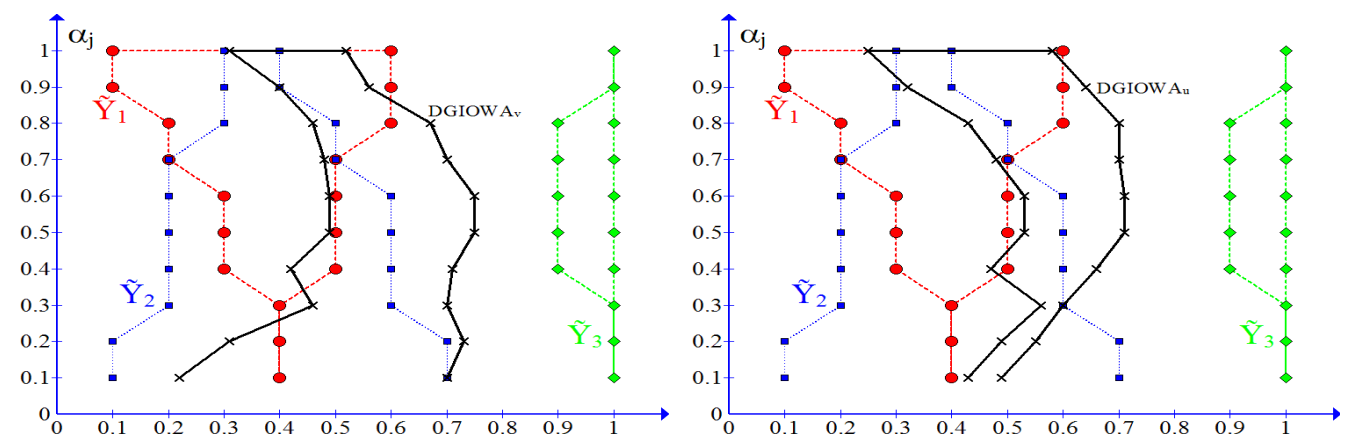

Fig. 3. Discrete gradual intervals $\widetilde{Y}_{1}, \widetilde{Y}_{2}, \widetilde{Y}_{3}$ and $\operatorname{DGIOW} A_{\widetilde{\mathbf{w}}}^{\stackrel{\complement_{A}^{\mathrm{A}, \mathbf{v}^{\prime}, g}}{\mathrm{~B}}}\left(\widetilde{Y}_{1}, \widetilde{Y}_{2}, \widetilde{Y}_{3}\right)$ for the weighting vector $\mathbf{v}$ (on the left)

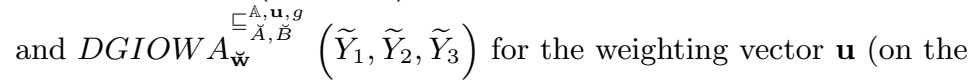
right), see Example 5.

- Making the information uniform: 11

DM choose:

- $k$ supervisors $s_{1}, \ldots, s_{k}$, possibly with different importance expressed by a weighting vector $\mathbf{v}=\left(v_{1}, \ldots, v_{k}\right) \in[0,1]^{k}$ with $v_{1} \leq \ldots \leq v_{k}, v_{1}+\ldots+v_{k}=1$,

- a weighting vector $\breve{\mathbf{w}}=\left(\breve{w}_{1}, \ldots, \breve{w}_{m}\right) \in D G_{k}([0,1])^{m}$ with $\breve{w}_{1}+\ldots+\breve{w}_{m}=\breve{1}$ which expresses the importance of particular criterions $c_{1}, \ldots, c_{m}$, to be more precise $\breve{w}_{i}$ expresses the importance of criterion with $i$ th largest assessment.

Each supervisor $s_{j}$ choose $m$ experts - one expert $e_{j i}$ for each criterion $c_{i}, j=$ $1, \ldots, k, i=1, \ldots, m$. Then each expert $e_{j i}$ assesses each alternative alt $t_{l}$ according to criterion $c_{i}$ by interval $\left[x_{j l i}^{-}, x_{j l i}^{+}\right] \subseteq[0,1], j=1, \ldots, k, i=1, \ldots, m, l=1, \ldots, n$. The following discrete gradual intervals are created from this assessment:

$$
\widetilde{X}_{l i}=\left[\breve{x}_{l i}^{-}, \breve{x}_{l i}^{+}\right]
$$

where $\breve{x}_{l i}^{-}\left(\alpha_{j}\right)=x_{j l i}^{-}, \breve{x}_{l i}^{+}\left(\alpha_{j}\right)=x_{j l i}^{+}$, i. e.,

$$
\widetilde{X}_{l i}\left(\alpha_{j}\right)=\left[x_{j l i}^{-}, x_{j l i}^{+}\right]
$$

for all $j=1, \ldots, k, i=1, \ldots, m, l=1, \ldots, n$, where $\alpha_{j}=j / k$.

The result of this phase is given by Table 6 .

Note that this phase gives more freedom in assessment, because the DM can make assessment without any constraints arising from the need of getting fuzzy sets (functions).

\footnotetext{
${ }^{1}$ We added this 'preparatory' phase before aggregation phase to show one possible way of obtaining experts' preferences in the form of discrete gradual intervals. Of course, this is not the only way, but we will not develop this point here. We leave for the future works the study of this problem.
} 


\begin{tabular}{c|c|c|c|c} 
& $c_{1}$ & $c_{2}$ & $\ldots$ & $c_{m}$ \\
\hline alt $_{1}$ & $\widetilde{X}_{11}$ & $\widetilde{X}_{12}$ & $\ldots$ & $\widetilde{X}_{1 m}$ \\
alt $_{2}$ & $\widetilde{X}_{21}$ & $\widetilde{X}_{22}$ & $\ldots$ & $\widetilde{X}_{2 m}$ \\
$\vdots$ & $\vdots$ & $\vdots$ & & $\vdots$ \\
alt $_{n}$ & $\widetilde{X}_{n 1}$ & $\widetilde{X}_{n 2}$ & $\ldots$ & $\widetilde{X}_{n m}$
\end{tabular}

Tab. 6. The result of the first phase. Each alternative alt $_{l}$ is assessed according to each criterion $c_{i}$ by discrete gradual interval $\widetilde{X}_{l i}$.

- Aggregation phase:

Choose $\mathbb{A}, g, \breve{A}, \breve{B}$ and calculate DGIOWA operators for each row of Table 6

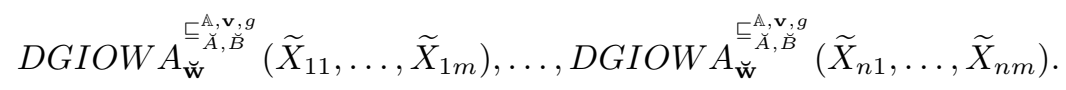

- Exploitation phase:

Take as solution the alternative corresponding to the largest DGIOWA according to linear order $\complement_{\breve{A}, \breve{B}}^{\mathbb{A}, \mathbf{v}, g}$.

Example 6. A hospital (DM) plans to buy an apparatus. There are three different kinds of apparatus $\left(\right.$ alt $_{1}$, alt $_{2}$, alt $\left._{3}\right)$ under consideration. The decision should be made with respect to three properties of apparatus $\left(c_{1}, c_{2}, c_{3}\right)$ in such a way that the apparatus would be appropriate for five different diagnosis $\left(d_{1}, \ldots, d_{5}\right)$ with importance expressed by weighting vector $\mathbf{v}=(0.1,0.15,0.2,0.25,0.3)$. The DM chooses a supervisor $\left(s_{1}, \ldots, s_{5}\right)$ for each diagnosis, each supervisor chooses three experts and each of the experts $\left(e_{j i}\right)$ assesses all kinds of apparatus from the point of view of particular criterion $\left(c_{i}\right)$ with respect to particular diagnosis $\left(d_{j}\right)$.

Let redistribution function $g$ be $g(i)=\frac{v_{i}}{1-v_{s}} \cdot v_{s}$, extended aggregation function $\mathbb{A}_{\mathbf{v}}$ be weighted average. Let aggregation function for discrete gradual real numbers $\breve{A}$ be induced by $K_{0.5}(a, b)=0.5 a+0.5 b$ and $\breve{B}$ be induced by $K_{0,6}(a, b)=0.6 a+0.4 b$ (note that $\breve{A}, \breve{B}$ satisfy the assumption of Theorem 3.5, see Lemma 3.6 and [3. Theorem 3.3]). Let the experts' assessments be given by Table 7 and $\breve{\mathbf{w}}=\left(\breve{w}_{1}, \breve{w}_{2}, \breve{w}_{3}\right)$ by Table 9 .

Then $\widetilde{X}_{11}, \ldots, \widetilde{X}_{33}$ arise by $14-15$ from the assessments, see Table 8 . Recall that this kind of assessment is not possible in other existing approaches [15, 31, because some $\widetilde{X}_{l i}$ are gradual intervals which are not functions (neither fuzzy sets), so, it is a specific benefit of using gradual intervals and our DGIOWA operator. From (11) we have:

$$
\widetilde{X}_{11} \sqsubseteq_{\breve{A}, \breve{B}}^{\mathbb{A}, \mathbf{v}, g} \widetilde{X}_{13} \sqsubseteq_{\breve{A}, \breve{B}}^{\mathbb{A}, \mathbf{v}, g} \widetilde{X}_{12}, \quad \widetilde{X}_{21} \sqsubseteq_{\breve{A}, \breve{B}}^{\mathbb{A}, \mathbf{v}, g} \widetilde{X}_{22} \sqsubseteq_{\breve{A}, \breve{B}}^{\mathbb{A}, \mathbf{v}, g} \widetilde{X}_{23}, \quad \widetilde{X}_{33} \sqsubseteq_{\breve{A}, \breve{B}}^{\mathbb{A}, \mathbf{v}, g} \widetilde{X}_{32} \sqsubseteq_{\breve{A}, \breve{B}}^{\mathbb{A}, \mathbf{v}, g} \widetilde{X}_{31} .
$$

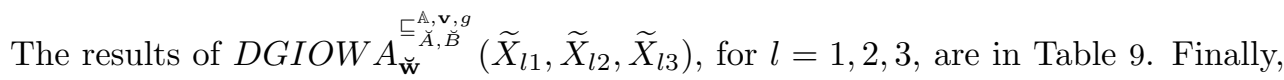
by (11) we have:

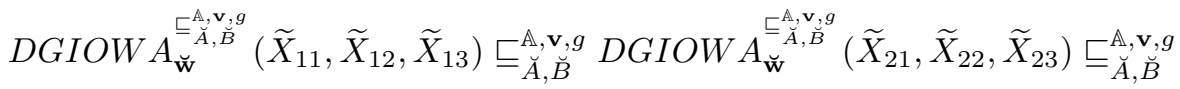

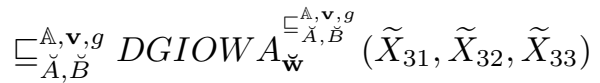


hence alt $_{3} \succ a l t_{2} \succ a l t_{1}$, where $a l t_{i} \succ$ alt $_{j}$ means that alternative alt $_{i}$ is better than alternative $a l t_{j}$. Thus the best alternative is alt $t_{3}$.

From Table 9 we can see that the weighting vector $\breve{\mathbf{w}}=\left(\breve{w}_{1}, \breve{w}_{2}, \breve{w}_{3}\right)$ puts stress on the largest gradual interval among $\widetilde{X}_{l 1}, \widetilde{X}_{l 2}, \widetilde{X}_{l 3}$ and only leaves slight influence to the smallest one. This has a major impact on the outcome. For instance, if $\breve{w}_{1}=\breve{w}_{2}=$ $\breve{w}_{3}=\frac{\breve{1}}{3}$, then the results would reversed: $a l t_{1} \succ a l t_{2} \succ a l t_{3}$, hence the best alternative would be alt 1 .

\begin{tabular}{|c|c|c|c|c|c|c|c|}
\hline & \multicolumn{3}{|c|}{$s_{1}$} & \multicolumn{3}{|c|}{$s_{2}$} & \\
\hline & $e_{11}$ & $e_{12}$ & $e_{13}$ & $e_{21}$ & $e_{22}$ & $e_{23}$ & \\
\hline alt $_{1}$ & {$[0.8,0.9]$} & {$[0.55,0.8]$} & {$[0.6,0.75]$} & {$[0.6,0.7]$} & {$[0.65,0.75]$} & {$[0.7,0.7]$} & \\
\hline alt $_{2}$ & {$[0.4,0.5]$} & {$[0.45,0.85]$} & {$[0.7,0.9]$} & {$[0.5,0.6]$} & {$[0.5,0.8]$} & {$[0.9,1]$} & $\ldots$ \\
\hline \multirow[t]{3}{*}{ alt $_{3}$} & {$[0.8,1]$} & {$[0.4,0.9]$} & {$[0.4,0.5]$} & {$[0.9,1]$} & {$[0.4,0.9]$} & {$[0.2,0.3]$} & \\
\hline & \multicolumn{3}{|c|}{$s_{3}$} & \multicolumn{3}{|c|}{$s_{4}$} & \\
\hline & $e_{31}$ & $e_{32}$ & $e_{33}$ & $e_{41}$ & $e_{42}$ & $e_{43}$ & \\
\hline alt $_{1}$ & {$[0.7,0.8]$} & {$[0.65,0.75]$} & {$[0.6,0.8]$} & {$[0.5,0.6]$} & {$[0.65,0.7]$} & {$[0.6,0.75]$} & \\
\hline alt $_{2}$ & {$[0.3,0.4]$} & {$[0.55,0.8]$} & {$[0.8,0.9]$} & {$[0.4,0.6]$} & {$[0.6,0.75]$} & {$[0.7,0.9]$} & $\ldots$ \\
\hline \multirow[t]{3}{*}{ alt $_{3}$} & {$[0.8,0.9]$} & {$[0.4,0.85]$} & {$[0.3,0.4]$} & {$[1,1]$} & {$[0.5,0.85]$} & {$[0.2,0.4]$} & \\
\hline & \multicolumn{3}{|c|}{$s_{5}$} & & & & \\
\hline & $e_{51}$ & $e_{52}$ & $e_{53}$ & & & & \\
\hline alt $_{1}$ & {$[0.6,0.7]$} & {$[0.7,0.7]$} & {$[0.6,0.8]$} & & & & \\
\hline alt $_{2}$ & {$[0.4,0.5]$} & {$[0.65,0.75]$} & {$[0.8,0.9]$} & & & & \\
\hline alt $_{3}$ & {$[0.9,1]$} & {$[0.5,0.8]$} & {$[0.3,0.5]$} & & & & \\
\hline
\end{tabular}

Tab. 7. The experts' assessments of the alternatives: expert $e_{j i}$, chosen by supervisor $s_{j}$, assesses criterion $c_{i}, j=1, \ldots, 5, i=1, \ldots, 3$

(Example 6).

\begin{tabular}{c|ccc|ccc|c}
$\alpha_{j}$ & $\widetilde{X}_{11}$ & $\widetilde{X}_{12}$ & $\widetilde{X}_{13}$ & $\widetilde{X}_{21}$ & $\widetilde{X}_{22}$ & $\widetilde{X}_{23}$ & \\
\hline 1 & {$[0.6,0.7]$} & {$[0.7,0.7]$} & {$[0.6,0.8]$} & {$[0.4,0.5]$} & {$[0.65,0.75]$} & {$[0.8,0.9]$} & \\
0.8 & {$[0.5,0.6]$} & {$[0.65,0.7]$} & {$[0.6,0.75]$} & {$[0.4,0.6]$} & {$[0.6,0.75]$} & {$[0.7,0.9]$} & \\
0.6 & {$[0.7,0.8]$} & {$[0.65,0.75]$} & {$[0.6,0.8]$} & {$[0.3,0.4]$} & {$[0.55,0.8]$} & {$[0.8,0.9]$} & $\ldots$ \\
0.4 & {$[0.6,0.7]$} & {$[0.65,0.75]$} & {$[0.7,0.7]$} & {$[0.5,0.6]$} & {$[0.5,0.8]$} & {$[0.9,1]$} & \\
0.2 & {$[0.8,0.9]$} & {$[0.55,0.8]$} & {$[0.6,0.75]$} & {$[0.4,0.5]$} & {$[0.45,0.85]$} & {$[0.7,0.9]$} & \\
\hline \hline$\alpha_{j}$ & $\widetilde{X}_{31}$ & $\widetilde{X}_{32}$ & $\widetilde{X}_{33}$ & & & \\
\hline 1 & {$[0.9,1]$} & {$[0.5,0.8]$} & {$[0.3,0.5]$} & & & \\
0.8 & {$[1,1]$} & {$[0.5,0.85]$} & {$[0.2,0.4]$} & & & \\
0.6 & {$[0.8,0.9]$} & {$[0.4,0.85]$} & {$[0.3,0.4]$} & & & \\
0.4 & {$[0.9,1]$} & {$[0.4,0.9]$} & {$[0.2,0.3]$} & & & \\
0.2 & {$[0.8,1]$} & {$[0.4,0.9]$} & {$[0.4,0.5]$} & & &
\end{tabular}

Tab. 8. The assessments: $\widetilde{X}_{l i}$ assess alternative alt $_{l}$ according to criterion $c_{i}$, see Example 6 . 


\begin{tabular}{c|ccc|ccc}
$\alpha_{j}$ & $\breve{w}_{1}$ & $\breve{w}_{2}$ & $\breve{w}_{3}$ & $D G I O W A_{1}$ & $D G I O W A_{2}$ & $D G I O W A_{3}$ \\
\hline 1 & 0.6 & 0.3 & 0.1 & {$[0.66,0.73]$} & {$[0.715,0.815]$} & {$[0.72,0.89]$} \\
0.8 & 0.6 & 0.3 & 0.1 & {$[0.62,0.705]$} & {$[0.64,0.825]$} & {$[0.77,0.895]$} \\
0.6 & 0.5 & 0.35 & 0.15 & {$[0.64,0.775]$} & {$[0.638,0.79]$} & {$[0.585,0.808]$} \\
0.4 & 0.5 & 0.35 & 0.15 & {$[0.66,0.725]$} & {$[0.7,0.87]$} & {$[0.62,0.86]$} \\
0.2 & 0.45 & 0.35 & 0.2 & {$[0.618,0.803]$} & {$[0.553,0.803]$} & {$[0.58,0.865]$}
\end{tabular}

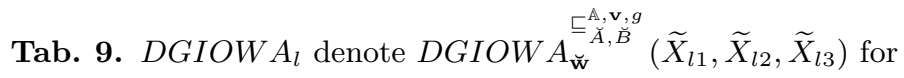
$l=1 \ldots, 3$ (Example 6$)$.

\section{CONCLUSION}

OWA operator is one of the most widely used aggregation method at present. The existence of a linear order of elements is a crucial point for applying OWA operators to the elements. This paper introduces a class of linear orders for discrete gradual real numbers and, based on the notion of admissible orders, also for discrete gradual intervals. From proposed linear order of discrete gradual intervals one can induce a linear order of fuzzy intervals with finite range. Moreover, the relation between the partial orders of gradual intervals and partial order of fuzzy intervals (membership grades of type-2 fuzzy sets) used in the type-2 fuzzy sets setting is described.

DGIOWA is an extension of IVOWA (OWA operator for intervals [3]) and 'standard' OWA operators. It is also proved that DGIOWA operator satisfies similar properties as 'standard' OWA operator (boundary conditions, monotonicity). Furthermore, the DGIOWA is also applicable to fuzzy intervals and then, for real weights, the aggregated value is fuzzy interval too.

An application of proposed linear orders and OWA operators to multi-expert decision making problem is shown.

\section{ACKNOWLEDGEMENT}

This work was partially supported by the Grant VEGA 1/0420/15.

(Received October 17, 2014)

\section{REFERENCES}

[1] G. Beliakov, A. Pradera, and T. Calvo: Aggregation Functions: A guide for practitioners. Studies in Fuzziness and Soft Computing 221 (2007), 261-269. DOI:10.1007/978-3-54073721-6_5

[2] H. Bustince, E. Barrenechea, T. Calvo, S. James, and G. Beliakov: Consensus in multiexpert decision making problems using penalty functions defined over a Cartesian product of lattices. Inform. Fusion 17 (2014), 56-64. DOI:10.1016/j.inffus.2011.10.002

[3] H. Bustince, J. Fernandez, A. Kolesárová, and R. Mesiar: Generation of linear orders for intervals by means of aggregation functions. Fuzzy Sets and Systems 220 (2013), 69-77. DOI:10.1016/j.fss.2012.07.015 
[4] H. Bustince, M. Galar, B. Bedregal, A. Kolesárová, and R. Mesiar: A new approach to interval-valued Choquet integrals and the problem of ordering in intervalvalued fuzzy set applications. IEEE Trans. Fuzzy Systems 21 (2013), 1150-1162. DOI:10.1109/tfuzz.2013.2265090

[5] O. Castillo and P. Melin: A review on interval type-2 fuzzy logic applications in intelligent control. Inform. Sciences 279 (2014), 615-631. DOI:10.1016/j.ins.2014.04.015

[6] F. Chiclana, F. Herrera, and E. Herrera-Viedma: Integrating three representation models in fuzzy multipurpose decision making based on fuzzy preference relations. Fuzzy Sets and Systems 97 (1998), 33-48. DOI:10.1016/s0165-0114(96)00339-9

[7] D. Dubois, E. Kerre, R. Mesiar, and H. Prade: Fuzzy interval analysis. In: Fundamentals of Fuzzy Sets (D. Dubois and H. Prade, eds.), The Handbooks of Fuzzy Sets Series, Vol. 7, Springer US 2000, pp. 483-581. DOI:10.1007/978-1-4615-4429-6_11

[8] D. Dubois and H. Prade: A review of fuzzy set aggregation connectives. Inform. Sciences 36 (1985), 85-121. DOI:10.1016/0020-0255(85)90027-1

[9] D. Dubois and H. Prade: Gradual elements in a fuzzy set. Soft Computing 12 (2008), 165-175. DOI:10.1007/s00500-007-0187-6

[10] J. Fortin, D. Dubois, and H. Fargier: Gradual numbers and their application to fuzzy interval analysis. IEEE Trans. Fuzzy Systems 16 (2008), 388-402. DOI:10.1109/tfuzz.2006.890680

[11] M. Grabisch, J. L. Marichal, R. Mesiar, and E. Pap: Aggregation Functions. Cambridge University Press, Cambridge 2009. DOI:10.1017/cbo9781139644150

[12] F. Herrera and L. Martínez: A model based on linguistic 2-tuples for dealing with multigranular hierarchical linguistic contexts in multi-expert decision-making. IEEE Trans. Systems Man and Cybernetics, Part B: Cybernetics 31 (2001), 227-234. DOI:10.1109/3477.915345

[13] N. N. Karnik and J. M. Mendel: Operations on type-2 fuzzy sets. Fuzzy Sets and Systems 122 (2001), 327-348. DOI:10.1016/s0165-0114(00)00079-8

[14] W. Kosiński, P. Prokopowicz, and A. Rosa: Defuzzification functionals of ordered fuzzy numbers. IEEE Trans. Fuzzy Systems 21 (2013), 1163-1169. DOI:10.1109/tfuzz.2013.2243456

[15] I. Lizasoain and C. Moreno: OWA operators defined on complete lattices. Fuzzy Sets and Systems 224 (2013), 36-52. DOI:10.1016/j.fss.2012.10.012

[16] W.A. Lodwick and E. A. Untiedt: A comparison of interval analysis using constraint interval arithmetic and fuzzy interval analysis using gradual numbers. In: Annual Meeting of the North American Fuzzy Information Processing Society, NAFIPS 2008, pp.1-6. DOI:10.1109/nafips.2008.4531302

[17] T. P. Martin and B. Azvine: The X-mu approach: Fuzzy quantities, fuzzy arithmetic and fuzzy association rules. In: IEEE Symposium on Foundations of Computational Intelligence (FOCI), 2013, pp. 24-29. DOI:10.1109/foci.2013.6602451

[18] P. Melin and O. Castillo: A review on type-2 fuzzy logic applications in clustering, classification and pattern recognition. Applied Soft Computing J. 21 (2014), 568-577. DOI:10.1016/j.asoc.2014.04.017

[19] R. Mesiar, A. Kolesárová, T. Calvo, and M. Komorníková: A review of aggregation functions. In: Fuzzy Sets and Their Extensions: Representation, Aggregation and Models (H. Bustince et al., eds.), Springer, Berlin 2008, pp. 121-144. DOI:10.1007/978-3-54073723-0_7 
[20] M. Mizumoto and K. Tanaka: Some properties of fuzzy sets of type 2. Information and Control 31 (1976), 312-340. DOI:10.1016/s0019-9958(76)80011-3

[21] E. R. Moore: Methods and Applications of Interval Analysis. SIAM 1979. DOI:10.1137/1.9781611970906

[22] E. R. Moore and W. A. Lodwick: Interval analysis and fuzzy set theory. Fuzzy Sets and Systems 135 (2003), 5-9. DOI:10.1016/s0165-0114(02)00246-4

[23] G. Ochoa, I. Lizasoain, D. Paternain, H. Bustince, and N. R. Pal: Some properties of lattice OWA operators and their importance in image processing. In: Proc. IFSAEUSFLAT 2015, pp. 1261-1265. DOI:10.2991/ifsa-eusflat-15.2015.178

[24] M. Roubens: Fuzzy sets and decision analysis. Fuzzy Sets and Systems 90 (1997), 199-206. DOI:10.1016/s0165-0114(97)00087-0

[25] D. Sánchez, M. Delgado, M. A. Vila, and J. Chamorro-Martínez: On a non-nested level-based representation of fuzziness. Fuzzy Sets and Systems 192 (2012), 159-175. DOI:10.1016/j.fss.2011.07.002

[26] Z. Takáč: Aggregation of fuzzy truth values. Inform. Sciences 271 (2014), 1-13. DOI:10.1016/j.ins.2014.02.116

[27] Z. Takáč: A linear order and OWA operator for discrete gradual real numbers. In: Proc. IFSA-EUSFLAT 2015, pp. 260-266. DOI:10.2991/ifsa-eusflat-15.2015.39

[28] C. L. Walker and E. A. Walker: The algebra of fuzzy truth values. Fuzzy Sets and Systems 149 (2005), 309-347. DOI:10.1016/j.fss.2003.12.003

[29] Z.S. Xu and Q. L. Da: The uncertain OWA operator. Int. J. Intelligent Systems 17 (2002), 569-575. DOI:10.1002/int.10038

[30] R. R. Yager: On ordered weighted averaging aggregation operators in multicriteria decisionmaking. IEEE Transactions on Systems Man and Cybernetics 18 (1988), 183-190. DOI:10.1109/21.87068

[31] S. M. Zhou, F. Chiclana, R. I. John, and J. M. Garibaldi: Type-1 OWA operators for aggregating uncertain information with uncertain weights induced by type-2 linguistic quantifiers. Fuzzy Sets and Systems 159 (2008), 3281-3296. DOI:10.1016/j.fss.2008.06.018

[32] S. M. Zhou, F. Chiclana, R. I. John, and J. M. Garibaldi: Alpha-level aggregation: A practical approach to type-1 OWA operation for aggregating uncertain information with applications to breast cancer treatments. IEEE Tran. Knowledge Data Engrg. 23 (2011), 1455-1468. DOI:10.1109/tkde.2010.191

Zdenko Takáč, Institute of Information Engineering, Automation and Mathematics, Faculty of Chemical and Food Technology, Slovak University of Technology in Bratislava, Radlinského 9, 812 37 Bratislava. Slovak Republic.

e-mail: zdenko.takac@stuba.sk 